\title{
A Cable-Driven Parallel Robot with an Embedded Tilt-Roll Wrist
}

\author{
Saman Lessanibahri \\ PhD student \\ École Centrale de Nantes, \\ Laboratoire des Sciences du \\ Numérique de Nantes, UMR 6004, \\ 1 Rue de la Noë, 44321, \\ Nantes, France \\ Email: Saman.Lessanibahri@Is2n.fr
}

\author{
Philippe Cardou \\ Professor, ASME Member \\ Laboratoire de robotique \\ Département de génie mécanique \\ Laboratoire des Sciences du \\ Numérique de Nantes, UMR 6004 \\ Université Laval \\ Québec, QC, Canada \\ Email: pcardou@gmc.ulaval.ca
}

\author{
Stéphane Caro* \\ Research Director, ASME Member \\ Centre National de la Recherche Scientifique \\ Laboratoire des Sciences du \\ Numérique de Nantes, UMR 6004 \\ 1 Rue de la Noë, 44321 \\ Nantes, France \\ Email: Stephane.Caro@Is2n.fr
}

\begin{abstract}
This paper addresses the optimum design, configuration and workspace analysis of a Cable-Driven Parallel Robot (CDPR) with an embedded tilt-roll wrist. The manipulator consists in a tilt-roll wrist mounted on the moving platform of a suspended CDPR. The embedded wrist provides large amplitudes of tilt and roll rotations and a large translational workspace obtained by the CDPR. This manipulator is suitable for tasks requiring large rotation and translation workspaces like tomography scanning, camera-orienting devices and visual surveillance. The movingplatform is an eight-degree-of-freedom articulated mechanism with large translational and rotational workspaces and it is suspended from a fixed frame by six cables. The manipulator employs two bi-actuated cables, i.e., cable loops to transmit the power from motors fixed on the ground to the tilt-roll wrist. Therefore, the manipulator achieves better dynamic performances due to a lower inertia of its moving-platform.
\end{abstract}

\footnotetext{
${ }^{*}$ Corresponding author
} 


\section{Introduction}

Cable-Driven Parallel Robots (CDPRs) consist of a base frame and a moving-platform connected to each other through cables. These cables are actuated by winches connected to motors that can vary cable tensions and lengths. When compared to classical parallel robots, CDPRs have advantages in terms of large translation workspace [1,2], heavy payload capacity [3], reconfigurability [4] and of performing high-speed tasks [5].

Despite their large translation workspaces, CDPRs are generally unable to provide large amplitudes of rotation of their moving-platform due to collisions between their moving parts. In general, the workspace of parallel robots can be increased by combining them with other parallel or serial mechanisms and constructing hybrid mechanisms. To the best of the authors' knowledge, there is but a limited number of papers addressing the question of extending the rotation workspaces of CDPRs. In [6], a parallel spherical wrist was introduced into the design of a CDPR to obtain large rotation and translation workspaces. The authors showed that the workspace of CDPRs can be enlarged by combining the advantages of the parallel spherical wrist in terms of rotation amplitudes with those of CDPRs in terms of large translation workspace.

Cable-loops or bi-actuated cable circuits are employed for different reasons in the design of several CDPRs, such as increasing the size of their wrench-feasible workspace [7-9]. Another purpose of cable-loops is the actuation of embedded end-effectors on the moving-platforms (hybrid mechanisms) through cable-loops and drums, to avoid the need of mounting motors on the moving-platform. In [10,11], two concepts of hybrid CDPRs were detailed. In [10], a hoist was combined with an under-constrained CDPR, where the large rotation amplitudes of the hoist were used to control the height of a payload while minimizing interferences with obstacles close to the ground. In the latter paper, the concept of a CDPR, with large translational workspace and large tilt and roll rotations of its end-effector was introduced. The main contributions of this paper with regard to [11] lie in: (i) a simplified kineto-static modeling of the mechanism at hand; (ii) the design optimization of a CDPR with an embedded Tilt-Roll wrist; (iii) the prototyping and experimentation. Hybrid mechanisms generally undergo reduced kinematic and dynamic performance due to their design complexity and high inertia of the moving-platform. However, by employing cable-loops into the design of hybrid CDPRs, these adverse effects are minimum.

Each cable-loop has two distinct purposes in the CDPR with an embedded tilt-roll wrist: positioning of the movingplatform and rotating an input bevel gear of the tilt-roll wrist. The cable-loops transmit power directly from motors attached on the ground to the articulated moving-platform. This contributes to lowering the inertia properties of the moving-platform and improving the dynamic performance of the manipulator.

Figure 1 shows the overall schematic of the manipulator and Fig. 2 represents the schematic of the moving-platform with the embedded tilt-roll wrist. Figure 3 illustrates the section-view of the moving-platform with its main components as 
$\mathscr{P}_{i}, i=1, \ldots, 5$. The proposed manipulator consists of the base frame, namely, $\mathscr{P}_{0}$, an under-constrained moving-platform, $\mathscr{P}_{1}$, which is suspended by eight cables. Two bi-actuated cables and four uni-actuated cables are illustrated in Fig. 1. Each cable loop forms a circuit by connecting two actuators while passing through two anchor points on the moving-platform and coiling about a gear on the tilt-roll wrist. Two motions can be induced by the cable-loop depending on the relative rotation of its two actuators. The first one is the displacement of the moving-platform for identical inputs to the two motors. The second motion is the rotation of the gears $\mathscr{P}_{3}$ and $\mathscr{P}_{4}$ about their respective axes $\left(z_{3}\right.$ and $\left.z_{4}\right)$ as shown in Fig. 2 , when the two actuators rotate in opposite directions.

An approach to the optimum design and configuration of the CDPR with an embedded tilt-roll wrist is divided into the following sections. Section 2 presents the kinetostatic model of the manipulator. Section 3 deals with the static workspace analysis of the manipulator at hand. The optimum design and cable configuration of the moving-platform are discussed in Sec. 4. Section 5 is about the prototyping, experimental validation and discussion about the obtained results on positioning accuracy. Finally, conclusions are drawn in Sec 6.

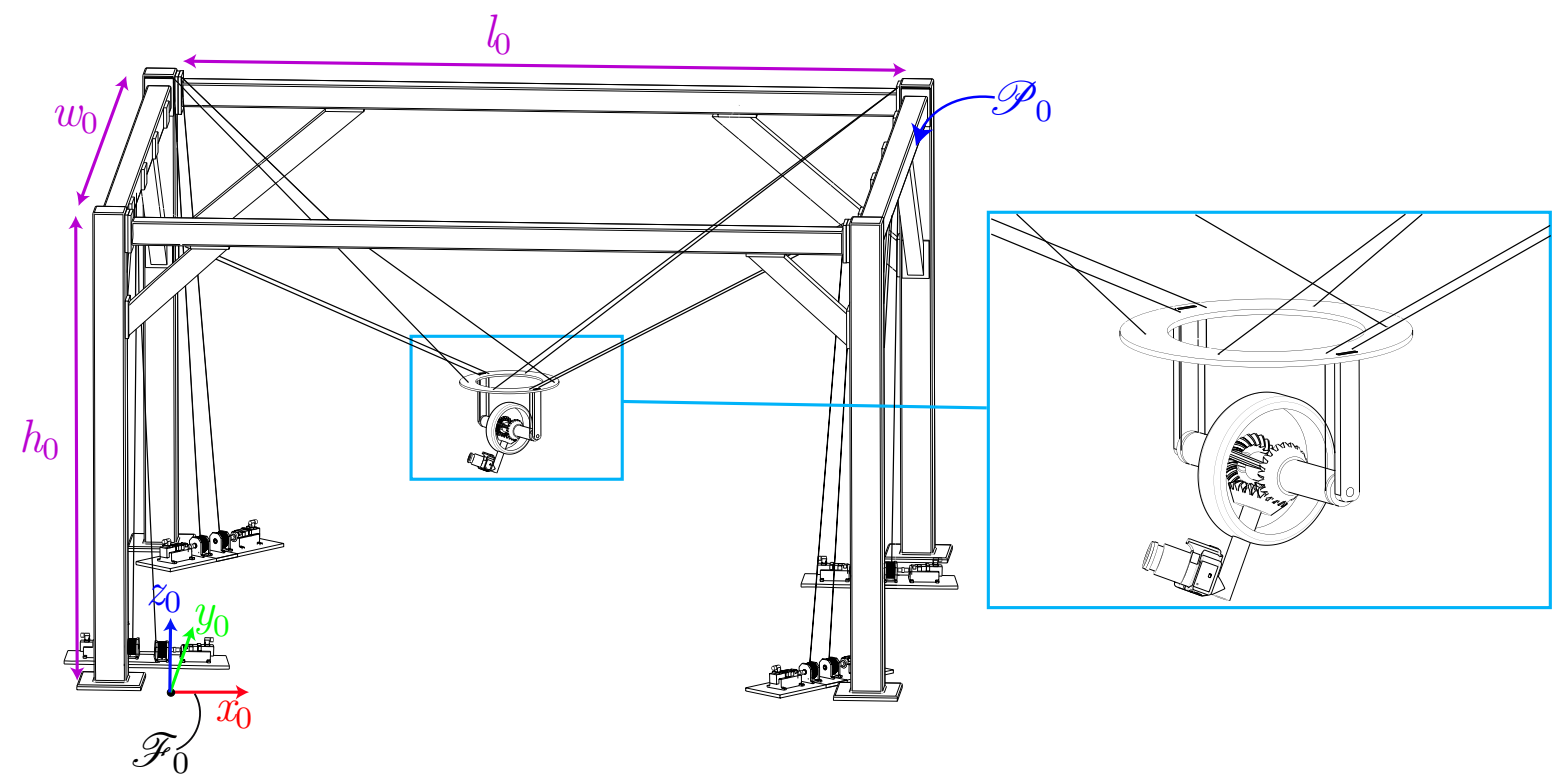

Fig. 1: Schematic of the manipulator 


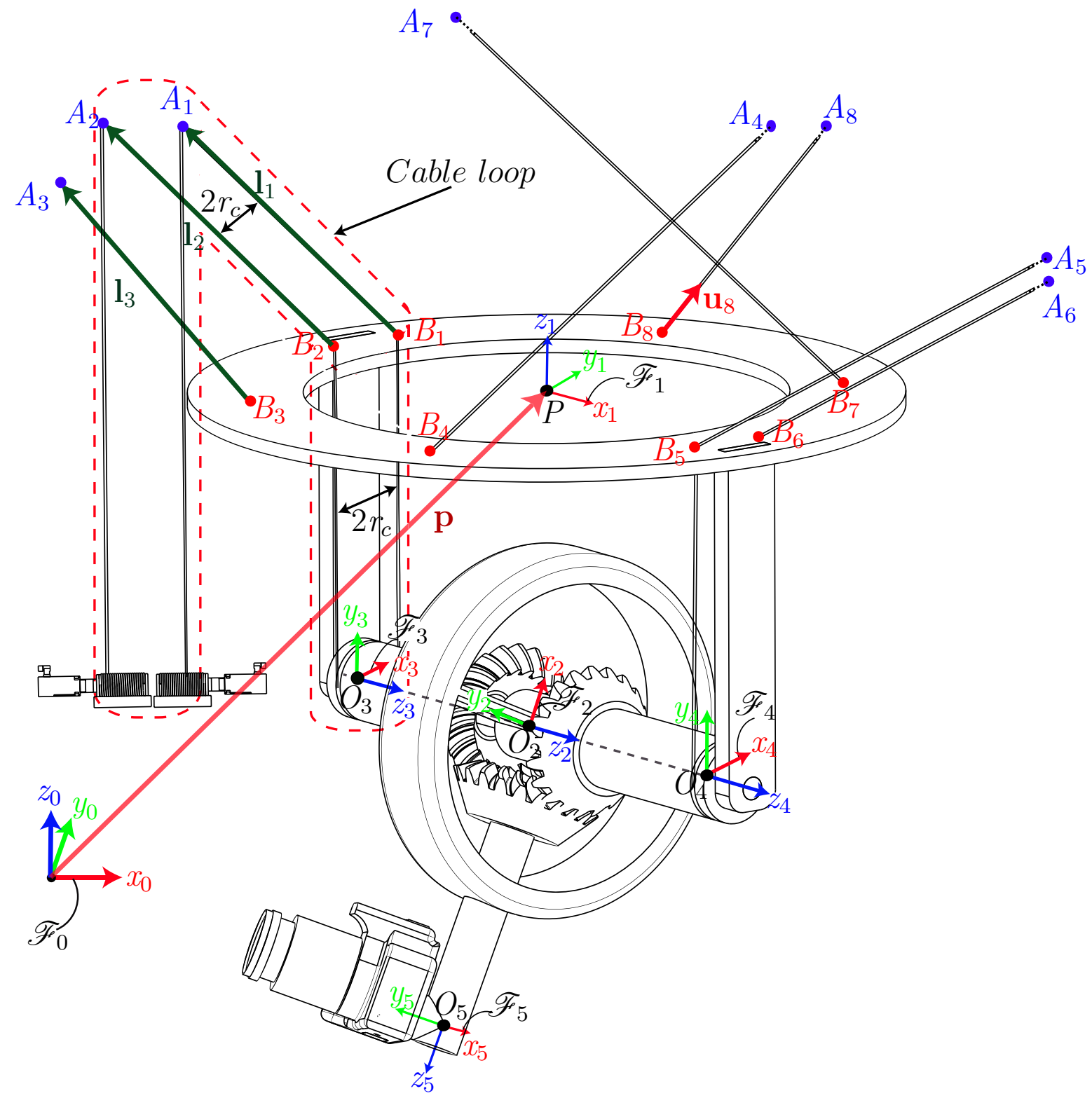

Fig. 2: Schematic of the moving-platform with an embedded tilt-roll wrist

\section{Kinetostatic Model of the Manipulator}

In this section, we present the kinetostatic model of the overall manipulator. In order to define the manipulator wrench matrix, we first introduce the loop-closure equations of the CDPR, which are given by:

$$
{ }^{0} \mathbf{l}_{i}={ }^{0} \mathbf{a}_{i}-{ }^{0} \mathbf{p}-{ }^{0} \mathbf{R}_{1}{ }^{1} \mathbf{b}_{i}, \quad i=1,2, \ldots, 8
$$




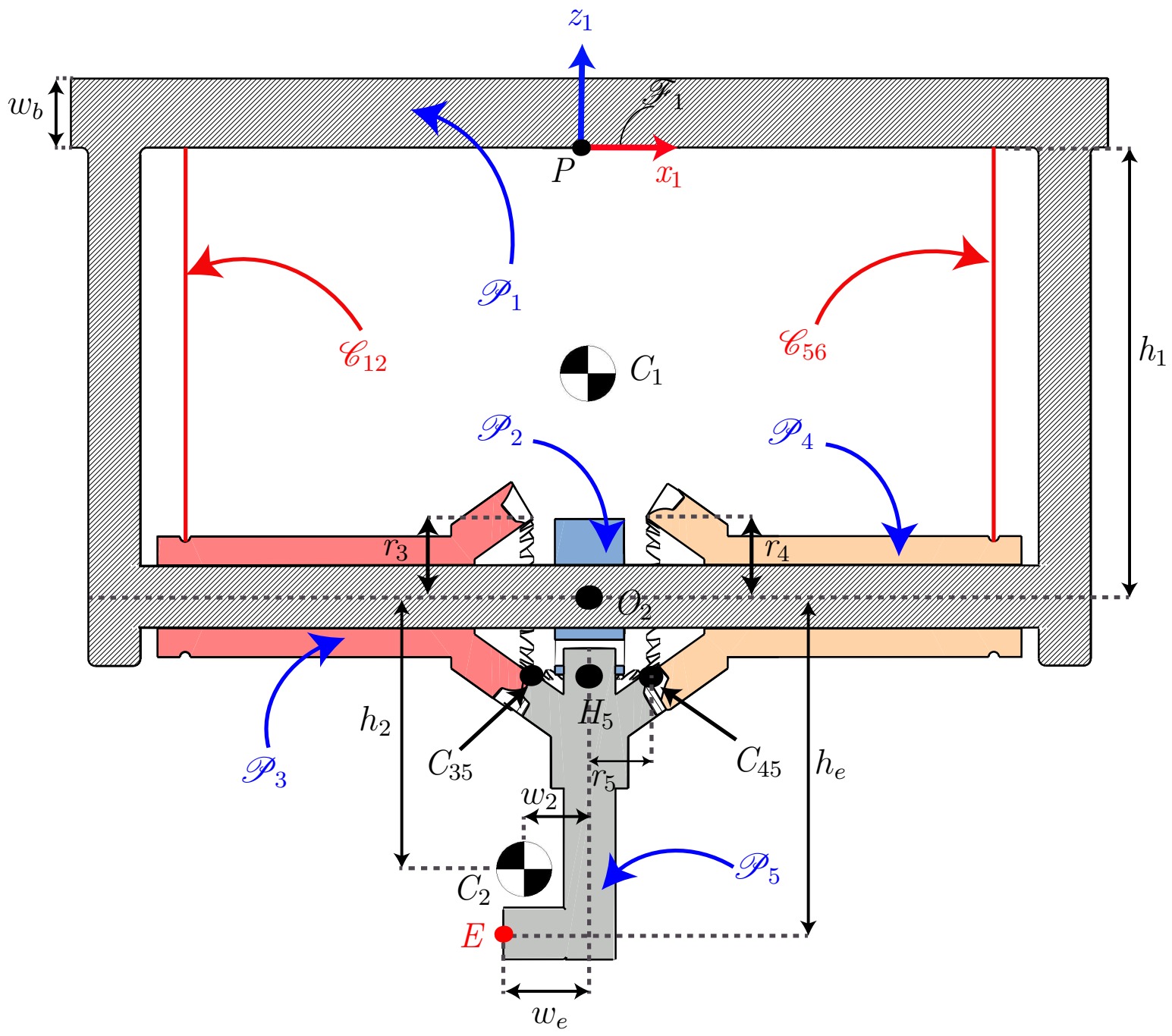

Fig. 3: Section-view of the moving-platform and the tilt-roll wrist

where ${ }^{0} \mathbf{l}_{i}$ is the $i$ th cable vector, i.e., the vector pointing from point $B_{i}$ to point $A_{i}$. Points $A_{i}$ and $B_{i}$ stand for the $i$ th cable exit point and anchor point, respectively. The former point is the location of the $i$ th pulley fixed to the ceiling and the latter is the connection point between the cable and the moving-platform. ${ }^{0} \mathbf{a}_{i},{ }^{1} \mathbf{b}_{i}$ and ${ }^{0} \mathbf{p}$ are the Cartesian coordinate vectors of points $A_{i}, B_{i}$ and $P$, respectively. ${ }^{0} \mathbf{R}_{1}$ is the rotation matrix from frame $\mathscr{F}_{0}$ to frame $\mathscr{F}_{1} \cdot \mathbf{t}_{i}, i=1,2, \ldots, 8$, stands for the $i$ th cable tension vector. $\mathbf{t}_{i}=t_{i}{ }^{0} \mathbf{u}_{i}$ and its magnitude is expressed as $t_{i}=\left\|\mathbf{t}_{i}\right\|_{2}, i=1, \ldots, 8 .{ }^{0} \mathbf{u}_{i}$ denotes the $i$ th cable unit vector namely,

$$
{ }^{0} \mathbf{u}_{i}=\frac{{ }^{0} \mathbf{l}_{i}}{l_{i}}, \quad i=1,2, \ldots, 8
$$

$l_{i}$ being the $i$ th cable length. 
The equilibrium of the external forces applied on the moving-platform is formulated as follows:

$$
\sum_{i=1}^{8} t_{i}^{0} \mathbf{u}_{i}+m \mathbf{g}=0
$$

where $m \mathbf{g}$ is the weight of the moving-platform. $m$ denotes total mass of the moving-platform and the spherical wrist. The equilibrium of moments applied onto the moving-platform about point $P$ expressed in frame $\mathscr{F}_{0}$ takes the form:

$$
\sum_{i=1}^{8} t_{i}\left({ }^{0} \mathbf{R}_{1}{ }^{1} \mathbf{b}_{i} \times{ }^{0} \mathbf{u}_{i}\right)+m\left({ }^{0} \mathbf{c}-{ }^{0} \mathbf{p}\right) \times{ }^{0} \mathbf{g}=0
$$

with $\mathbf{c}$ being the Cartesian coordinates vector of the Center of Mass (CoM) of the moving-platform:

$$
\mathbf{c}=\frac{m_{1} \mathbf{c}_{1}+m_{2} \mathbf{c}_{2}}{m_{1}+m_{2}}
$$

$\mathbf{c}_{1}$ is the Cartesian coordinates vector of the CoM of components $\mathscr{P}_{1}$ to $\mathscr{P}_{4}$ with mass of $m_{1}$ defined as:

$$
m_{1}=m_{b}+2 m_{c}+m_{s}+m_{\mathscr{P}_{2}}+m_{\mathscr{P}_{3}}+m_{\mathscr{P}_{4}}
$$

$m_{b}, m_{c}$ and $m_{s}$ being the masses of the moving-platform components i.e., its base, columns and shaft, respectively. $\mathbf{c}_{2}$ is the Cartesian coordinates vector of the CoM of the terminal link $\mathscr{P}_{5}$, its mass being equal to $m_{2}$. Therefore, the total mass of the moving-platform is expressed as follows:

$$
m=m_{1}+m_{2}
$$


The input tilt-roll wrist torques $\tau_{3}$ and $\tau_{4}$ are functions of the cable tension difference in cable-loops $\mathscr{C}_{12}$ and $\mathscr{C}_{56}$, respectively:

$$
\begin{aligned}
& \tau_{3}=r_{c}\left(t_{1}-t_{2}\right) \\
& \tau_{4}=r_{c}\left(t_{6}-t_{5}\right)
\end{aligned}
$$

$r_{c}$ being the radius of the groove made in $\mathscr{P}_{3}$ and $\mathscr{P}_{4}$ to house the two cable-loops. From Eqs. (3) to (9), the static equilibrium model of the manipulator is expressed in a matrix form as:

$$
\mathbf{W t}+\mathbf{w}_{g}=\mathbf{0}_{8}
$$

where $\mathbf{0}_{8}$ is the eight-dimensional zero vector and the wrench matrix $\mathbf{W}$ takes the following form:

$$
\mathbf{W}=\left[\begin{array}{cccccccc}
{ }^{0} \mathbf{u}_{1} & { }^{0} \mathbf{u}_{2} & { }^{0} \mathbf{u}_{3} & { }^{0} \mathbf{u}_{4} & { }^{0} \mathbf{u}_{5} & { }^{0} \mathbf{u}_{6} & { }^{0} \mathbf{u}_{7} & { }^{0} \mathbf{u}_{8} \\
{ }^{0} \mathbf{d}_{1} & { }^{0} \mathbf{d}_{2} & { }^{0} \mathbf{d}_{3} & { }^{0} \mathbf{d}_{4} & { }^{0} \mathbf{d}_{5} & { }^{0} \mathbf{d}_{6} & { }^{0} \mathbf{d}_{7} & { }^{0} \mathbf{d}_{8} \\
r_{c} & -r_{c} & 0 & 0 & -r_{c} & r_{c} & 0 & 0 \\
\mu r_{c} & -\mu r_{c} & 0 & 0 & \mu r_{c} & -\mu r_{c} & 0 & 0
\end{array}\right]
$$

$\mu=\frac{r_{5}}{r_{3}}=\frac{r_{5}}{r_{4}}$ is the gear train ratio and the pitch radius of the $i$ th gear is denoted as $r_{i}$ and $i=3,4,5$. Besides,

$$
{ }^{0} \mathbf{d}_{i}={ }^{0} \mathbf{R}_{1}{ }^{1} \mathbf{b}_{i} \times{ }^{0} \mathbf{u}_{i}, \quad i=1, \ldots, 8 .
$$

The last two rows of $\mathbf{W}$ correspond to the static model of the tilt-roll wrist as presented in ref. [11]. $\mathbf{t}$ is the vector of tensions 
in the eight cables.

$$
\mathbf{t}=\left[\begin{array}{llllllll}
t_{1} & t_{2} & t_{3} & t_{4} & t_{5} & t_{6} & t_{7} & t_{8}
\end{array}\right]^{T}
$$

$\mathbf{w}_{g}$ is the eight-dimensional gravity wrench vector applied on the moving-platform and the tilt-roll wrist, namely,

$$
\mathbf{w}_{g}=\left[m^{0} \mathbf{g}^{T} \quad m\left({ }^{0} \mathbf{R}_{1}{ }^{1} \mathbf{c} \times{ }^{0} \mathbf{g}\right)^{T} \mathbf{m}_{\alpha, \beta}^{T}\right]^{T}
$$

where, ${ }^{0} \mathbf{g}=[0,0,-g]^{T}$ with $g=9.81 \mathrm{~m} / \mathrm{s}^{2}$. The Cartesian coordinates vector of $C_{1}$ expressed in $\mathscr{F}_{1}$ is denoted as

$$
{ }^{1} \mathbf{c}_{1}=\left[0,0, c_{1 z}\right]^{T}
$$

with,

$$
c_{1 z}=\frac{-h_{1}\left(m_{c}+m_{s}+m_{\mathscr{P}_{2}}+m_{\mathscr{P}_{3}}+m_{\mathscr{P}_{4}}\right)}{m_{1}}
$$

and variable Cartesian coordinates vector of $\mathrm{CoM}$, namely, $C_{2}$ is a function of $\alpha$ and $\beta$ as follows:

$$
{ }^{1} \mathbf{c}_{2}=\left[\begin{array}{c}
-w_{2} \sin \beta \\
-h_{2} \cos \alpha-w_{2} \sin \alpha \cos \beta \\
-h_{2} \sin \alpha+w_{2} \cos \alpha \cos \beta-h_{1}
\end{array}\right]
$$

The tilt angle of the end-effector is denoted as $\alpha=\angle\left(y_{1}, x_{2}\right)$ and its roll angle is defined as $\beta=\angle\left(z_{2}, x_{5}\right) . \mathbf{m}_{\alpha, \beta}$ is the array 
of the moments applied by gravity onto the tilt-roll wrist about axes $z_{2}$ and $z_{5}$, respectively:

$$
\mathbf{m}_{\alpha, \beta}=\left[m_{\alpha}, m_{\beta}\right]^{T}
$$

with $m_{\alpha}=m_{g_{z}}$ and $m_{\beta}=-m_{g_{x}}$.

The moment due to the mass of $\mathscr{P}_{5}$ exerted on the tilt-roll wrist expressed in $\mathscr{F}_{2}$ is denoted as:

$$
{ }^{2} \mathbf{m}_{g}=m_{2}\left({ }^{2} \mathbf{c}_{2} \times{ }^{2} \mathbf{R}_{0}^{0} \mathbf{g}\right)=m_{2} g\left[\begin{array}{c}
-w_{2} \sin \beta \cos \alpha \\
w_{2} \sin \beta \sin \alpha \\
h_{2} \cos \alpha+w_{2} \cos \beta \sin \alpha
\end{array}\right]
$$

\section{Workspace Analysis}

In this section, we introduce the static workspace of the CDPR with an embedded tilt-roll wrist. In general, the static workspace of the eight-DoF CDPR with an embedded tilt-roll wrist consists of the set of positions and orientations of the moving-platform and the orientations of the end-effector, namely, ${ }^{0} \mathbf{p}$ and ${ }^{0} \mathbf{R}_{1}$ and $\mathbf{q}_{T R}=[\alpha, \beta]^{T}$, which satisfies the static equilibrium of the manipulator. The set $\mathscr{T}$ represents the cable tension space and takes the shape of a box in an eightdimensional space:

$$
\mathscr{T}=\left\{\mathbf{t} \in \mathbb{R}^{8}: \mathbf{t}_{\min } \leq \mathbf{t} \leq \mathbf{t}_{\max }\right\}
$$

where, $\mathbf{t}_{\min }$ and $\mathbf{t}_{\max }$ are the cable tension limit vectors. The Static Workspace $\mathscr{S}$ is the set of the moving-platform poses and tilt-roll wrist configurations satisfying the static equilibrium of the manipulator under the action of gravity.

$$
\mathscr{S}=\left\{\left({ }^{0} \mathbf{p},{ }^{0} \mathbf{R}_{1}, \mathbf{q}_{T R}\right) \in \mathbb{R}^{3} \times S O(3) \times \mathbb{R}^{2}: \exists \mathbf{t} \in \mathscr{T}, \mathbf{W} \mathbf{t}+\mathbf{w}_{g}=\mathbf{0}_{8}\right\}
$$


where $S O(3)$ is the group of proper-orthogonal $3 \times 3$ matrices. As the visualization of such an eight-dimensional workspace is impossible with common human perception in $3 \mathrm{D}$, we define the static workspace of the manipulator for two different cases. From Eq. (21), we derive two subsets $\mathscr{S}_{1}$ and $\mathscr{S}_{2}$ based on the constrained orientations of the moving-platform and the tilt-roll wrist. The former subset, namely, $\mathscr{S}_{1}$ is a set for a given orientation of the moving-platform and the wrist, i.e., the translational SW of the manipulator with constant orientation of ${ }^{0} \mathbf{R}_{1}$ and $\mathbf{q}_{T R}$.

$$
\mathscr{S}_{1}=\left\{{ }^{0} \mathbf{p} \in \mathbb{R}^{3} \mid{ }^{0} \mathbf{R}_{1}=\mathbf{I}_{3}, \alpha=\beta=\pi / 2: \exists \mathbf{t} \in \mathscr{T}, \mathbf{W t}+\mathbf{w}_{g}=\mathbf{0}_{8}\right\}
$$

The minimum moment due to weight of $\mathscr{P}_{5}$ exerted on the tilt-roll wrist corresponds to $\alpha=\beta=\pi / 2$. Therefore, for largest possible workspace of $\mathscr{S}_{1}$ we consider those angles for tilt and roll of the wrist. $\mathscr{S}_{2}$ is defined as the static workspace of the manipulator for a constant orientation of the moving-platform while the tilt and roll angles are free to rotate.

$$
\mathscr{S}_{2}=\left\{{ }^{0} \mathbf{p} \in \mathbb{R}^{3} \mid{ }^{0} \mathbf{R}_{1}=\mathbf{I}_{3}:-\pi \leq \alpha \& \beta \leq \pi: \exists \mathbf{t} \in \mathscr{T}, \mathbf{W t}+\mathbf{w}_{g}=\mathbf{0}_{8}\right\}
$$

In order to trace the static workspace, the index, namely, the capacity margin index is employed to determine the static equilibrium of the moving-platform at a given pose. In [12-15] the capacity margin index was introduced and implemented for tracing wrench feasible workspace and static equilibrium of the CDPRs. In the latter papers, the algorithm of calculation of capacity margin is detailed. The foregoing method has been used in this paper to define and trace the workspaces of CDPRs with an embedded Tilt-Roll wrist, namely, a hybrid manipulator.

Hereafter, the capacity margin index can be checked to indicate whether a pose of the moving-platform belongs to the static workspace or not. By discretizing of the Cartesian space and interpolating between the index values, we are able to 
trace the static workspace. The discretization of Cartesian space is detailed in the following.

$$
\begin{aligned}
& x_{i}=x_{0}+i \delta \quad\left(i=0, \ldots, n_{x}\right) \\
& y_{j}=y_{0}+j \delta \quad\left(j=0, \ldots, n_{y}\right) \\
& z_{k}=z_{0}+k \delta \quad\left(k=0, \ldots, n_{z}\right)
\end{aligned}
$$

with $\delta$ being the resolution of the discretization and $\mathbf{o}_{0}=\left[x_{0}, y_{0}, z_{0}\right]^{T}$ denoting the origin of frame $\mathscr{F}_{0}$. The number of discretized points along $x, y$ and $z$ are given by

$$
\begin{aligned}
& n_{x}=\left\lfloor\frac{l_{0}-x_{0}}{\delta}\right\rfloor \\
& n_{y}=\left\lfloor\frac{w_{0}-y_{0}}{\delta}\right\rfloor \\
& n_{z}=\left\lfloor\frac{h_{0}-z_{0}}{\delta}\right\rfloor
\end{aligned}
$$

where, $l_{0}, w_{0}$ and $h_{0}$ are the length, width and height of the manipulator frame $\left(\mathscr{P}_{0}\right)$, respectively. The number of points found to be inside the static workspace, $\mathscr{S}_{1}$ and $\mathscr{S}_{2}$, are denoted as $N_{\mathscr{S}_{1}}$ and $N_{\mathscr{S}_{2}}$ as follows:

$$
\begin{aligned}
& N_{\mathscr{S}_{1}}=\left|\left\{\mathbf{p}\left(x_{i}, y_{j}, z_{k}\right) \in \mathscr{S}_{1}\right\}\right| \\
& N_{\mathscr{S}_{2}}=\left|\left\{\mathbf{p}\left(x_{i}, y_{j}, z_{k}\right) \in \mathscr{S}_{2}\right\}\right|
\end{aligned}
$$

with $x_{i}, y_{i}$ and $z_{i}$ being defined in Eqs. (24-26). The ratios of static workspace, namely $\mathscr{R}_{\mathscr{S}_{i}}, i=1,2$, is introduced to quantify the proportion of $\mathscr{S}_{1}$ and $\mathscr{S}_{2}$ to the task space, respectively.

$$
\begin{aligned}
\mathscr{R}_{\mathscr{S}_{1}} & =\frac{N_{\mathscr{S}_{1}}}{\left(n_{x}+1\right)\left(n_{y}+1\right)\left(n_{z}+1\right)} \\
\mathscr{R}_{\mathscr{S}_{2}} & =\frac{N_{\mathscr{S}_{2}}}{\left(n_{x}+1\right)\left(n_{y}+1\right)\left(n_{z}+1\right)}
\end{aligned}
$$




\section{Optimum Design of the Manipulator}

This section deals with the optimization of the CDPR design with an embedded tilt-roll wrist. The investigation of the optimum manipulator focuses on two goals. The first one is to determine the optimum cable arrangement. The second goal is to find the optimum design variables of the moving-platform. The design variables appearing in Table 1 are searched for whereas the design parameters given in Table 2 are predefined and constant. The optimization problem aims to maximize the volume of the static workspace, $\mathscr{R}_{\mathscr{S}_{2}}$. The decision variables describe the overall dimensions of the moving-platform while those of the tilt-roll wrist are considered as design parameters. The positions of the anchor points $B_{i}, i=1, \ldots, 8$ on $\mathscr{P}_{1}$ are also considered as decision variables in order to determine the optimum cable arrangement of the CDPR.

\section{Objective Function}

The cable-loops and the tilt-roll wrist have significant effects on the size and shape of the static workspace. The main goal of the manipulator is to achieve large amplitudes of rotations $\alpha$ and $\beta$ of its moving-platform across its workspace. Therefore, maximizing $\mathscr{R}_{\mathscr{S}_{2}}$ is the objective function considered to improve the performance of the manipulator.

\section{Design Variables}

All the exit-points of the CDPR, namely, $A_{i}$ and $i=1, \ldots, 8$ are located on the top of $\mathscr{P}_{0}$ and on its rectangle vertices. The vertices, namely, $\mathscr{A}_{i}$ and $i=1,2,3,4$ are illustrated in Fig. 4. Moreover, we formulate all the combinations of the cable arrangement by assuming that each $\mathscr{A}_{i}, i=1,2,3,4$ accommodates two exit-points. Therefore, cable $\mathscr{C}_{i}$ connects $A_{i}$ to $B_{i}$, and $i=1, \ldots, 8$. The total number $\mathscr{N}_{a}$ of cable arrangements is computed as:

$$
\mathscr{N}_{a}=\frac{P(n, k)}{v_{1} ! v_{2} ! v_{3} ! v_{4} !}=\frac{8 !}{(2 !)^{4}}=2520
$$

$P(n, k)$ refers to $k$-permutations of $n$ with $n=k=8$. The number of times that $\mathscr{A}_{i}, i=1,2,3,4$ can be chosen as exit points is noted as $v_{i}=2$. From Eq. (34), 2520 cable arrangements are considered for the CDPR under study. Decision variable, $\eta$ is associated to the cable arrangement of the CDPR.

The geometric design variables are shown in Figs. 3 and 4. The first decision variable, $r_{b}$, is the radius of the circle passing through $B_{i}, i=1, \ldots, 8 . h_{1}$ denotes the height of the moving-platform. $w_{b}$ is the width of the top-plate of the moving-platform. All the anchor points are located on a circle drawn on the top-plate with radius of $r_{b}$, as shown in Fig. 4. 


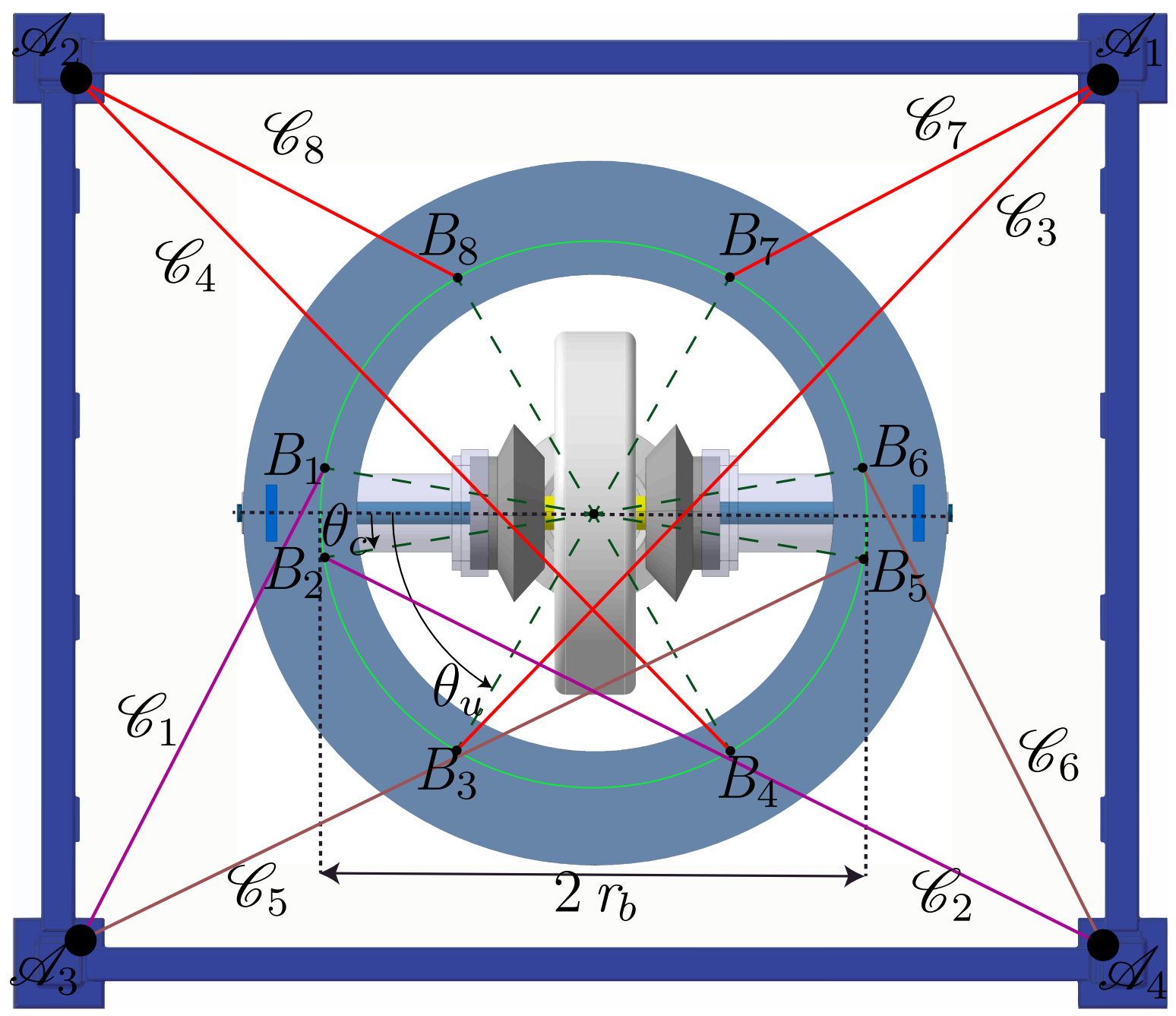

Fig. 4: Top-view of the base frame and up-scaled moving-platform with the embedded tilt-roll wrist

Therefore, the positions of the anchor points ${ }^{1} \mathbf{b}_{i}$ are defined in polar coordinates as follows:

$$
{ }^{1} \mathbf{b}_{i}=-r_{b}\left[\begin{array}{c}
\cos \theta_{i} \\
\sin \theta_{i}
\end{array}\right] \quad i=1, \ldots, 8
$$

The anchor point $B_{i}$ is connected to the exit point $A_{i}, i=1, \ldots, 8$. The exit points of the manipulator, $A_{i}$, are fixed on the top vertices of $\mathscr{P}_{0}$ as shown in Fig. 1.

The anchor points of the cable-loops, namely, $B_{1}, B_{2}, B_{5}, B_{6}$ are constrained to be close to $x_{1}$-axis in order to facilitate the actuation of $\mathscr{P}_{3}$ and $\mathscr{P}_{4}$. This constraint prevents undesired cable-loop routing. Moreover, the anchor points of the 
cable-loops are dependent on one another to guarantee the symmetric actuation of the tilt-roll wrist as follows:

$$
\begin{aligned}
& \theta_{1}=-\theta_{c} \\
& \theta_{2}=\theta_{c} \\
& \theta_{5}=\pi-\theta_{c} \\
& \theta_{6}=\pi+\theta_{c}
\end{aligned}
$$

$\theta_{c}$ is the cable loop angle depicted in Fig. 4.

Moreover, some constraints are defined to keep a symmetry of the anchor points $B_{i}$ onto the moving-platform. The following expresses the constraints relating the anchor points of uni-actuated cables to one another:

$$
\begin{aligned}
& \theta_{3}=\theta_{u} \\
& \theta_{4}=\pi-\theta_{u} \\
& \theta_{7}=\pi+\theta_{u} \\
& \theta_{8}=-\theta_{u}
\end{aligned}
$$

$\theta_{u}$ being the angle defining the location of the anchor points of the uni-actuated cables onto the moving-platform. Therefore, the positions of anchor points $B_{i}, i=1, \ldots, 8$, are functions of $\theta_{c}$ or $\theta_{u}$, and the vector $\mathbf{x}$ of decision variables becomes

$$
\mathbf{x}=\left[r_{b}, h_{1}, w_{b}, \theta_{c}, \theta_{u}, \eta\right]^{T}
$$

\section{Constraints}

The first constraint is set to prevent collision between the terminal link and the top-plate, $\mathscr{P}_{5}$ and $\mathscr{P}_{1}$, respectively. Point $E$ located on the tip of $\mathscr{P}_{5}$ is prone to undesired contact with $\mathscr{P}_{1}$. Hence, for the given parameters of the wrist $\left(h_{e}\right.$ and 
Table 1: Boundaries and optimum values of the design variables

\begin{tabular}{l|llllll}
\hline \hline Design variable & $r_{b}$ & $h_{1}$ & $w_{b}$ & $\theta_{c}$ & $\theta_{u}$ & $\eta$ \\
Unit & {$[\mathrm{mm}]$} & {$[\mathrm{mm}]$} & {$[\mathrm{mm}]$} & {$[\mathrm{deg}]$} & {$[\mathrm{deg}]$} & {[]} \\
\hline Lower-bound (lb) & 5 & 100 & 3 & -45 & 0 & 1 \\
Optimum value & 183 & 384 & 8 & 45 & 90 & 201 \\
Upper-bound (ub) & 210 & 400 & 10 & 45 & 360 & 2520 \\
\hline \hline
\end{tabular}

$w_{e}$ ), the full tilt rotation without any collision with the top-plate is guaranteed as long as:

$$
h_{e}^{2}+w_{e}^{2}<h_{1}^{2}
$$

The actuation of the tilt-roll wrist requires a tension difference between the two ends of a cable-loop. This tension difference is a function of the total mass $m$ of the tilt-roll wrist. The larger $m$, the higher the tension difference. Therefore, the upper bound $m_{\max }$ of $m$ is set to $5 \mathrm{~kg}$, i.e., $m_{\max }=5 \mathrm{~kg}$. The lower bound vector, $\mathbf{x}_{l b}$, and upper bound vector, $\mathbf{x}_{u b}$, of the decision variable vector $\mathbf{x}$ are given in Table 1.

Finally, the optimization problem at hand is formulated as follows:

$$
\begin{array}{ll}
\operatorname{maximize} & f(\mathbf{x})=\mathscr{R}_{\mathscr{S}_{2}} \\
\text { over } & \mathbf{x}=\left[r_{b}, h_{1}, w_{b}, \theta_{c}, \theta_{u}, \eta\right]^{T}
\end{array}
$$

subject to:

\section{Results}

$$
\begin{gathered}
g_{1}: h_{e}{ }^{2}+w_{e}{ }^{2}<h_{1}{ }^{2} \\
g_{2}: m<m_{\max } \\
\mathbf{x}_{l b} \leq \mathbf{x} \leq \mathbf{x}_{u b}
\end{gathered}
$$

The optimization problem is solved by using a genetic algorithm, i.e., with the ${ }^{C}$ Matlab $g a$ function. The algorithm begins by creating a random initial population. The algorithm then creates a sequence of new populations. At each step, the algorithm uses the individuals in the current generation to create the next population. To create the new population, the algorithm performs different steps including: (i) scoring each member of the current population by computing its fitness 
value; (ii) selecting members, called parents, based on their fitness value; (iii) producing children from the parents, children are produced either by making random changes to a single parent-mutation or by combining the vector entries of a pair of parents-crossover; $(i v)$ replacing the current population with the children to form the next generation. Finally, the algorithm stops when one of the stopping criteria is met. The tuning parameters of the algorithm are given in Tab. 3.

The obtained optimum design variable values are given in Tab. 1. The optimum design, cable configuration of the overall manipulator and its maximal static workspaces, namely $\mathscr{S}_{1}$ and $\mathscr{S}_{2}$, are illustrated in Fig. 5. The optimum design and cable configuration of the moving-platform yield 0.57 and 0.45 for $\mathscr{R}_{\mathscr{S}_{1}}$ and $\mathscr{R}_{\mathscr{S}_{2}}$, respectively.

$\mathscr{S}_{2}$ is the translation static workspace of the moving-platform for all possible orientations of the tilt-roll wrist.

Figure 6 shows the isocontours of ratio $\mathscr{R}_{\mathscr{S}_{2}}$ as a function of masses $m_{1}$ and $m_{2}$, with the decision variables set to their optimum values, except $r_{b}$ and $w_{b}$. Note that $m_{1}$ depends on $r_{b}, w_{b}$ and the material density $\rho . \rho=7800 \mathrm{~kg} / \mathrm{m}^{3}$ as the top plate is made up of steel. $\mathscr{R}_{\mathscr{S}_{2}}$ increases with $m_{1}$, while, on the contrary, it decreases with $m_{2}$. However, it can be noticed that the effect of $m_{1}$ on $\mathscr{R}_{\mathscr{S}_{2}}$ is negligible compared to that of $m_{2}$. The maximum applied moment onto $\mathscr{P}_{3}$ and $\mathscr{P}_{4}$ by cable-loops is proportional to the tension difference in the two segments of cable-loops. It is understood from the obtained results that the variable gravitational wrench onto the moving-platform induced by $\mathscr{P}_{5}$ mass has a significant impact on the size of the manipulator workspace.

Similarly, Figs. 7 to 9 illustrate the effect of $r_{b}, \theta_{c}$ and $\theta_{u}$ on $\mathscr{R}_{\mathscr{S}_{2}}$. From Figs. 8 to 9 , it is noteworthy that $\mathscr{R}_{\mathscr{S}_{2}}$ is very sensitive to variations in angle $\theta_{u}$. Indeed, a small variation in $\theta_{u}$ from its nominal value may lead to a significant decrease of $\mathscr{R}_{\mathscr{S}_{2}}$.

Table 2: Design parameters

\begin{tabular}{|c|c|c|}
\hline Parameter & Abbreviation & Value \\
\hline Mass of $\mathscr{P}_{2}[\mathrm{~g}]$ & $m_{\mathscr{P}_{2}}$ & 60 \\
\hline Mass of $\mathscr{P}_{3}[\mathrm{~g}]$ & $m_{\mathscr{P}_{3}}$ & 200 \\
\hline Mass of $\mathscr{P}_{4}[\mathrm{~g}]$ & $m_{\mathscr{P}_{4}}$ & 200 \\
\hline Mass of the terminal link $[\mathrm{g}]$ & $m_{2}$ & 600 \\
\hline Length of the terminal link [mm] & $h_{e}$ & 126 \\
\hline Width of terminal link $[\mathrm{mm}]$ & $w_{e}$ & 20 \\
\hline Minimum admissible cable tension $[\mathrm{N}]$ & $t_{\min }$ & 0 \\
\hline Maximum admissible cable tension $[\mathrm{N}]$ & $t_{\max }$ & 128 \\
\hline Gear ratio of the wrist [] & $\mu$ & 1 \\
\hline
\end{tabular}

Figure 10 shows the workspaces $\mathscr{W}_{1}$ and $\mathscr{W}_{2}$ of the classical CDPR obtained by replacing the two bi-actuated cables 
Table 3: Tuning parameters of the genetic algorithm

\begin{tabular}{lll}
\hline \hline Parameter & Abbreviation & Value \\
\hline Maximum Number of Generations & MNG & 100 \\
Function Tolerance & FT & $1 \times 10^{-4}$ \\
Population Size & PS & 200 \\
Crossover Fraction & CF & 0.8 \\
Elite Count & EC & $0.05 \times$ PS \\
\hline \hline
\end{tabular}

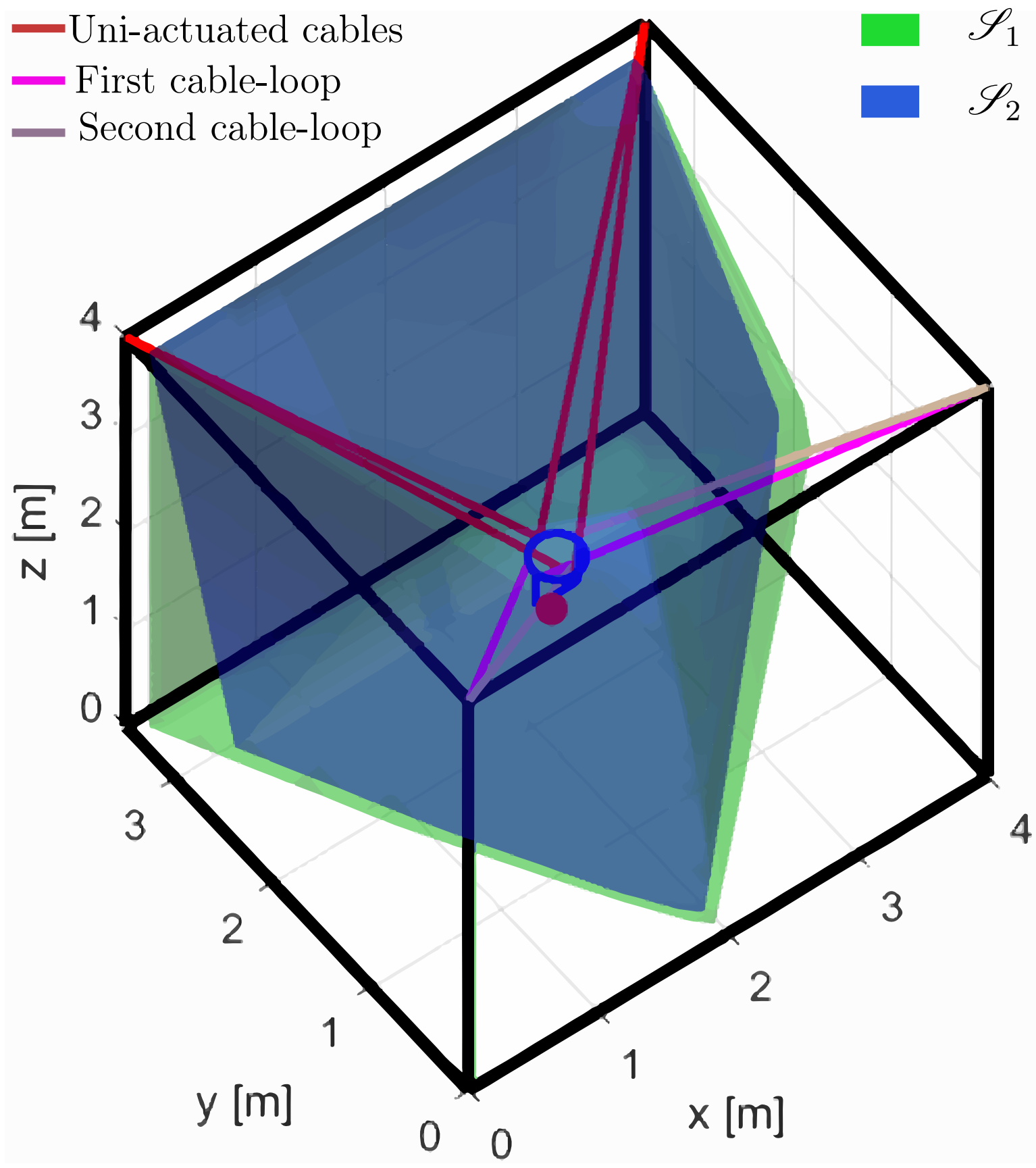

Fig. 5: Workspaces $\mathscr{S}_{1}$ and $\mathscr{S}_{2}$ associated with the optimum design of the CDPR with an embedded tilt-roll wrist 


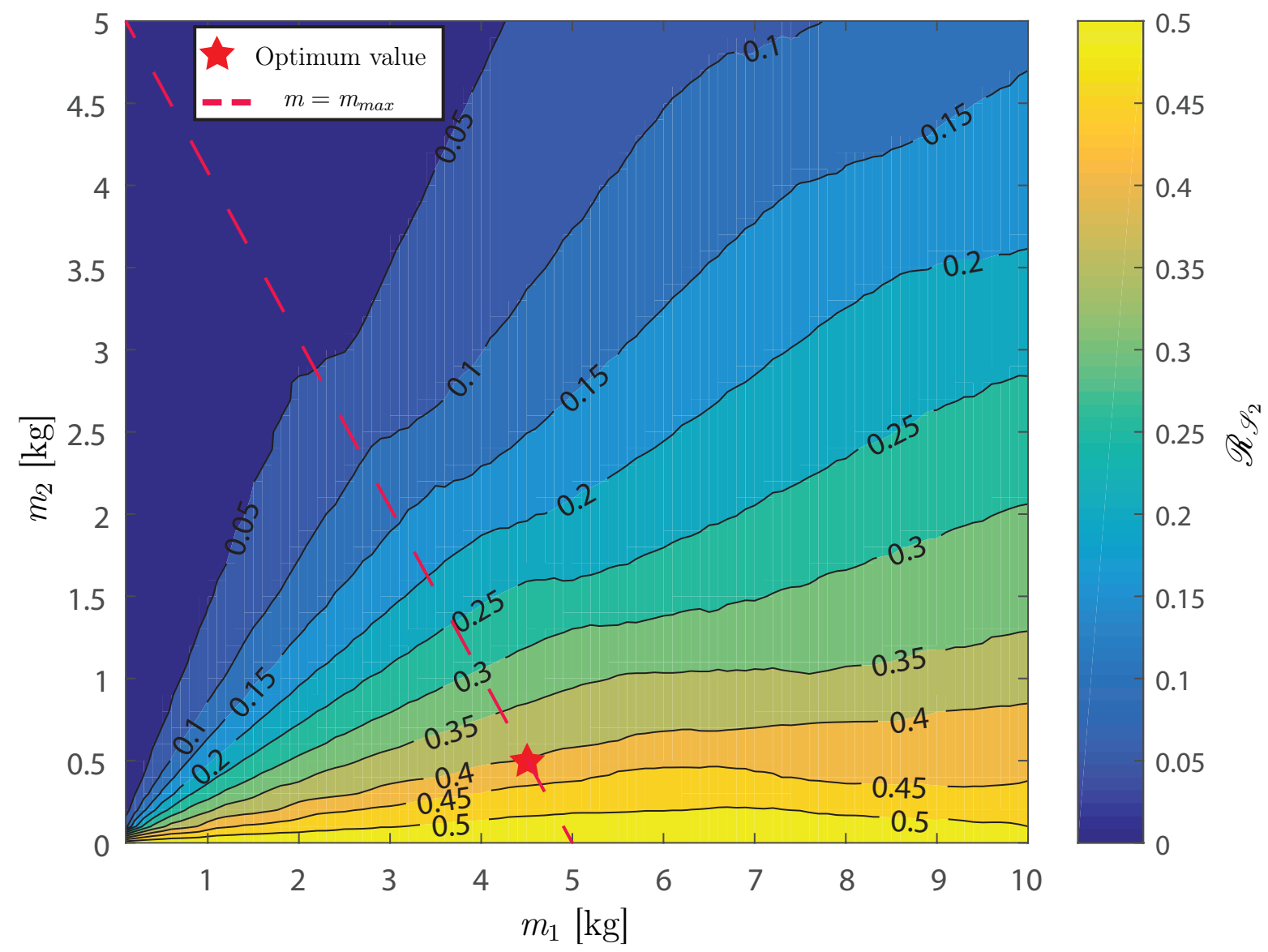

Fig. 6: Ratio $\mathscr{R}_{\mathscr{S}_{2}}$ of static workspace $\mathscr{S}_{2}$ as a function of $m_{1}$ and $m_{2}$, the other decision variables being set to their optimal values (Table 1)

(cable loops) depicted in Fig. 5 by four uni-actuated cables. $\mathscr{W}_{1}$ amounts to the static workspace of the corresponding classical CDPR with the gravity wrench defined as in (22). $\mathscr{W}_{2}$ amounts to the wrench-feasible workspace of the corresponding classical CDPR with the set of external wrenches due the tilt-roll wrist motion defined as in (23). From Figs. 5 and 10, it should be noted by substituting uni-actuated cables with bi-actuated cables (cable-loops), the size of the static workspace decreases. In spite of that, the moving-platform can reach large amplitudes of the tilt and roll rotations as a result of cableloops. It appears to be a trade-off between large translation and orientation workspaces due to the cable-loops in the fullyactuated CDPR. It is noteworthy that translation and orientation workspaces are maximal when employing two additional actuators as shown in Fig. 11.

\section{Prototyping and Experimentation}

The prototyping of the moving-platform with embedded tilt-roll wrist is presented in this section. The base frame of the CREATOR platform $\mathscr{P}_{0}$ is $4 \mathrm{~m}$ long $\left(l_{0}\right), 3.5 \mathrm{~m}$ wide $\left(w_{0}\right)$ and $4 \mathrm{~m}$ high $\left(h_{0}\right)$ as shown in Fig. 1 . Steel, aluminum alloys and ABS are used in the manufacturing of the prototype. The prototype of the moving-platform has the overall dimensions 


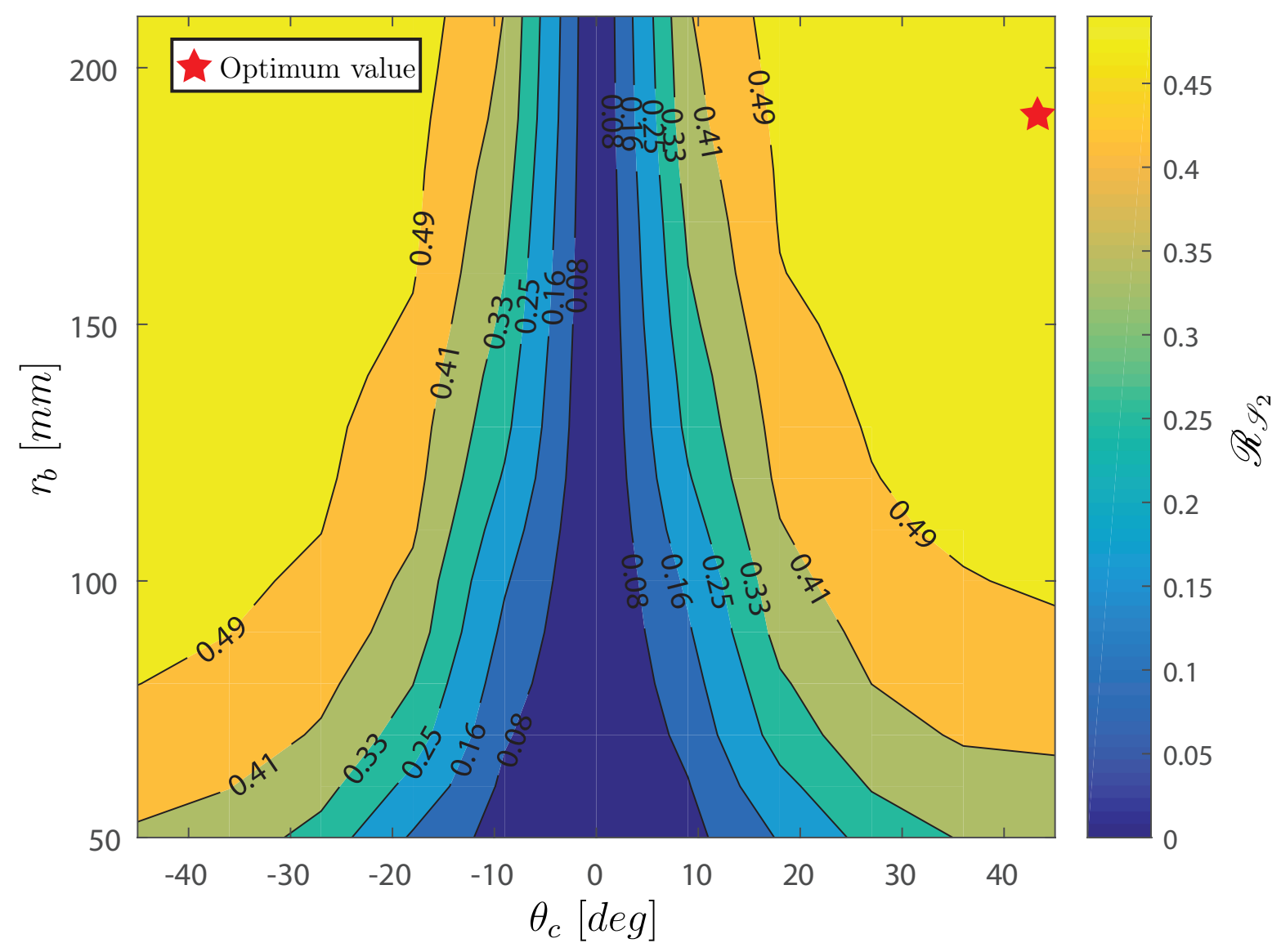

Fig. 7: Ratio $\mathscr{R}_{\mathscr{S}_{2}}$ of static workspace $\mathscr{S}_{2}$ as a function of $\theta_{c}$ and $r_{b}$, the other decision variables being set to their optimal values (Table 1)

of $20 \times 20 \times 25 \mathrm{~cm}$, as shown in Fig. 12. The masses of the components of the moving-platform are given in Table 2. The overall mass of the moving-platform is equal to $3.5 \mathrm{~kg}$. Note that a gearless tilt-roll wrist similar to the gearless pitch-roll wrist introduced in [16] could also be used.

This section also elaborates experimentation process of the CDPR with an embedded tilt-roll wrist. Firstly, we introduce the overall architecture of the demonstrator as depicted in Fig 13. The figure briefly demonstrates the main hardware of the manipulator as well as their connection to one another.

In order to evaluate the large orientation workspace and pose accuracy of the manipulator, two different test-trajectories are proposed. The first one shows the contribution of this CDPR in terms of its rotation workspace with large amplitudes. While, the second path-trajectory puts emphasize on the accuracy of the moving-platform and its sensitivity for a potential surveillance task.

The first test-trajectory ${ }^{1}$ focuses on the generation of a desired trajectory for the CDPR with tilt-roll wrist. The main aim of this trajectory is a straightforward demonstration of the large rotational workspace capability of the manipulator. The

\footnotetext{
${ }^{1}$ A video can be found at http://tiny.cc/oaiycz
} 


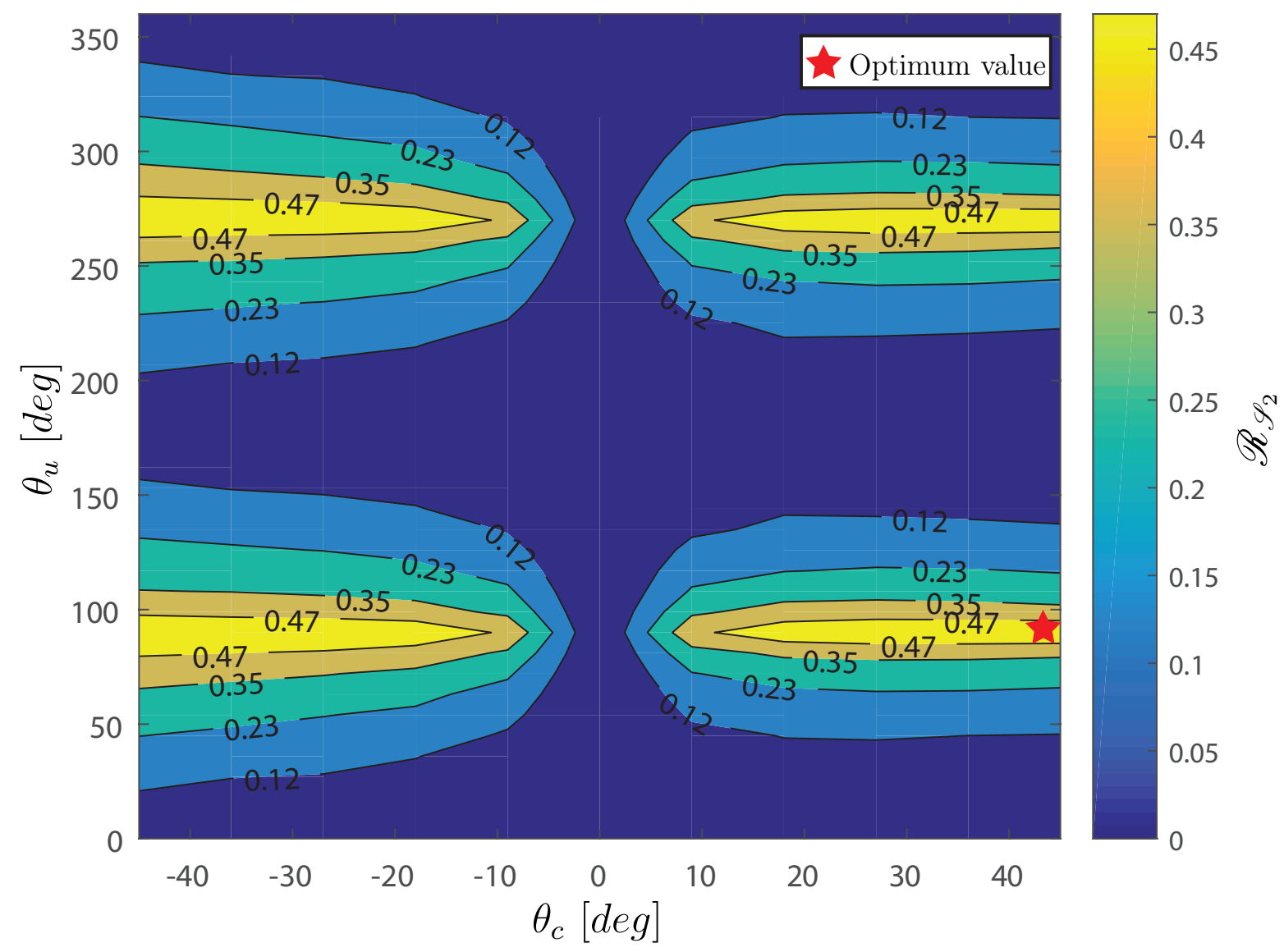

Fig. 8: Ratio $\mathscr{R}_{\mathscr{S}_{2}}$ of static workspace $\mathscr{S}_{2}$ as a function of $\theta_{c}$ and $\theta_{u}$, the other decision variables being set to their optimal values (Table 1)

test-trajectory is composed of several via-points such that the moving-platform translates between them based on a fifthorder polynomial profile of motions. Within via-points the tilt-roll wrist performs pure tilt, pure roll and combination of both motions. The amplitude of the rotation for the tilt and roll is $8 \pi$ radians, i.e., four turns.

The second test-trajectory is proposed to demonstrate one of the potential tasks of the CDPR with an embedded tiltroll wrist requiring high precision of the manipulator in position and orientation. For this task, a laser pointer is attached to the end-effector of the moving-platform. Overall, the laser dot aims at a fixed target on the ground while the movingplatform follows a circular path. The trajectory is designed to evaluate the precision and sensitivity of the tilt-roll wrist. The test-trajectory at hand ${ }^{2}$ consists of the following steps:

1. Start from the home-configuration of the moving-platform and proceed to the starting configuration of the circular path.

2. Perform the circular path of the end-effector while laser-pointer is aimed at the fixed target on the ground.

3. Return to the home-configuration of the moving-platform.

There is a disparity between the desired and actual trajectories during the second phase of the second test-trajectory

${ }^{2}$ A video can be found at http: //tiny.cc/cohycz 


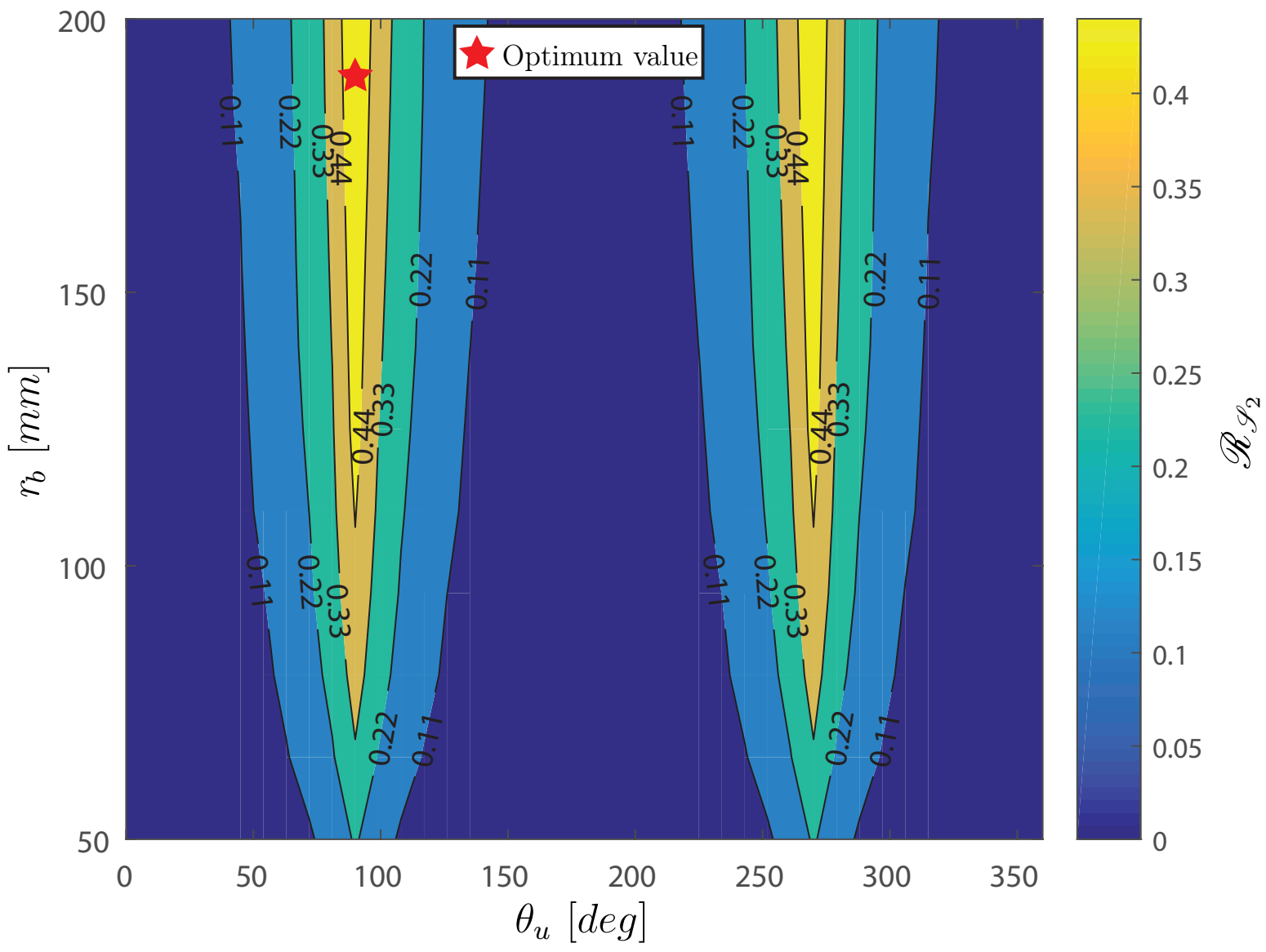

Fig. 9: Ratio $\mathscr{R}_{\mathscr{S}_{2}}$ of static workspace $\mathscr{S}_{2}$ as a function of $\theta_{u}$ and $r_{b}$, the other decision variables being set to their optimal values (Table 1)

as shown in Fig. 14. The figure presents the reconstruction of the laser dot through image processing. The upper-bound of the error in the position of the laser-dot with respect to the center of the target is around $30 \mathrm{~cm}$. There are several factors involved in the orienting error of the wrist, but the most influential seems to be the sensitivity of the tilt-roll wrist inputs. Figure 15 presents the variations of the cable lengths as a function of time. More specifically, Fig. 15a presents the cable length variations corresponding to all motions involved in the trajectory, while Fig. $15 \mathrm{~b}$ presents cable length variations corresponding only to wrist rotations. The maximum required cable length variation is not more than $15 \mathrm{~mm}$. This sensitivity can be reduced simply by increasing the gear ratio of the wrist, namely, $\mu$, which requires substantial modifications in the wrist design. Another solution to improve accuracy in control of the wrist is to employ more sophisticated cable models, e.g. an elastostatic model and a hysteresis model [17]. Moreover a better accuracy could be obtained by improving the control system and acquiring visual feedback on the pose of the manipulator $[18,19]$.

Figure 16 shows the proportion of the cable loop length variation required for $\alpha$ and $\beta$ to the maximum cable loop length variation during thorough test-trajectory 2. Clearly, the lengths of the cable loops are not limiting factors in our current design. In general, their lengths can be chosen to be large without affecting other characteristics of the CDPR. 


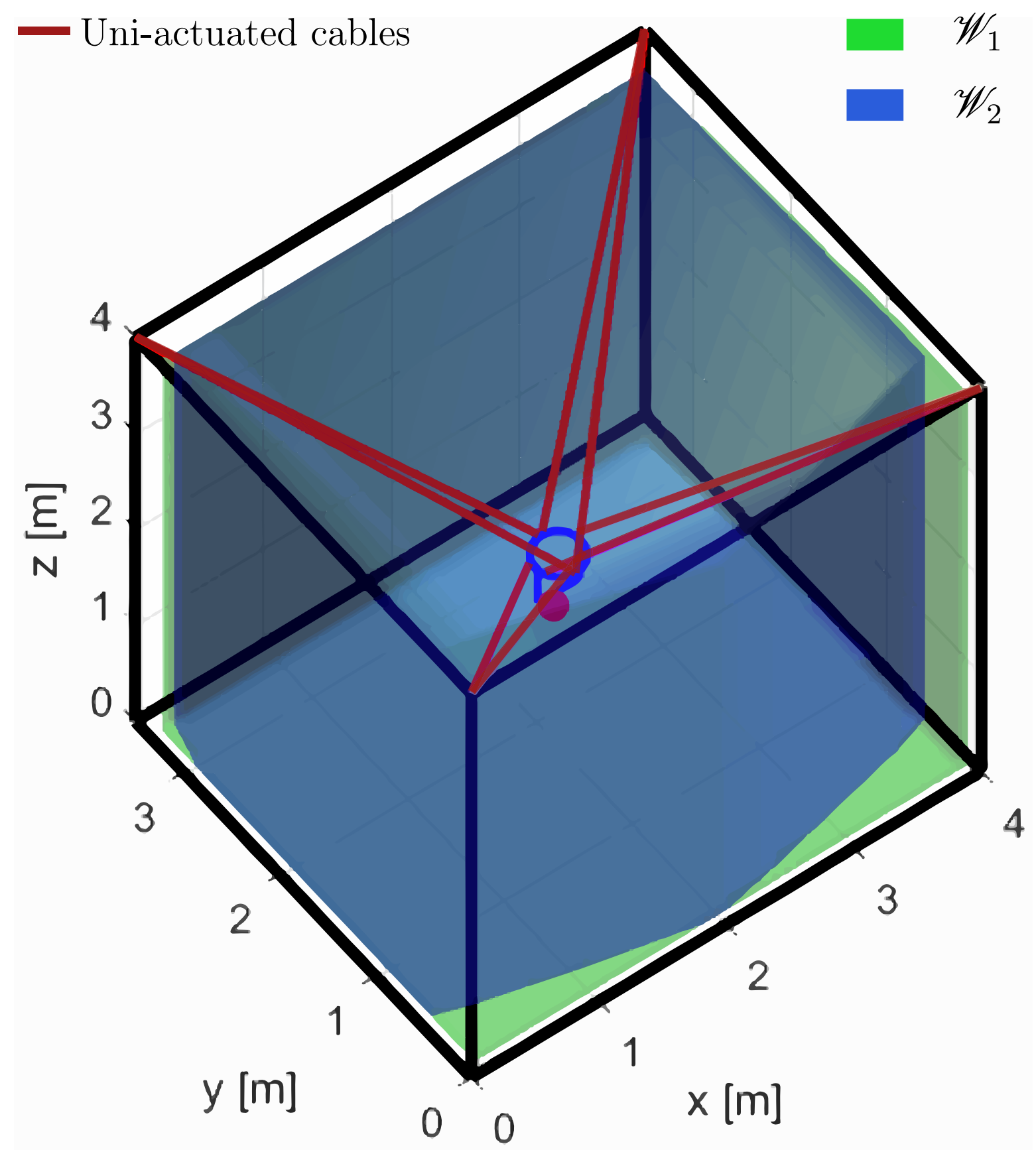

Fig. 10: Workspaces $\mathscr{W}_{1}$ and $\mathscr{W}_{2}$ of a classical CDPR with design variables values set to the optimum values given in Tab. 1

\section{Conclusions}

This paper addressed the optimum design and cable configuration of a Cable-Driven Parallel Robot (CDPR) with an embedded tilt-roll wrist for large translational and rotational workspaces. The eight-degree-of-freedom hybrid robot was studied in terms of its kinetostatic workspace. Moreover, design and prototyping of the CDPR with the embedded tilt-roll wrist was presented. The optimization results revealed that, the size of the static workspace highly relies on the specifications of the tilt-roll wrist. That is to say, the variable gravitational moment of the wrist and cable-loops arrangement have crucial 


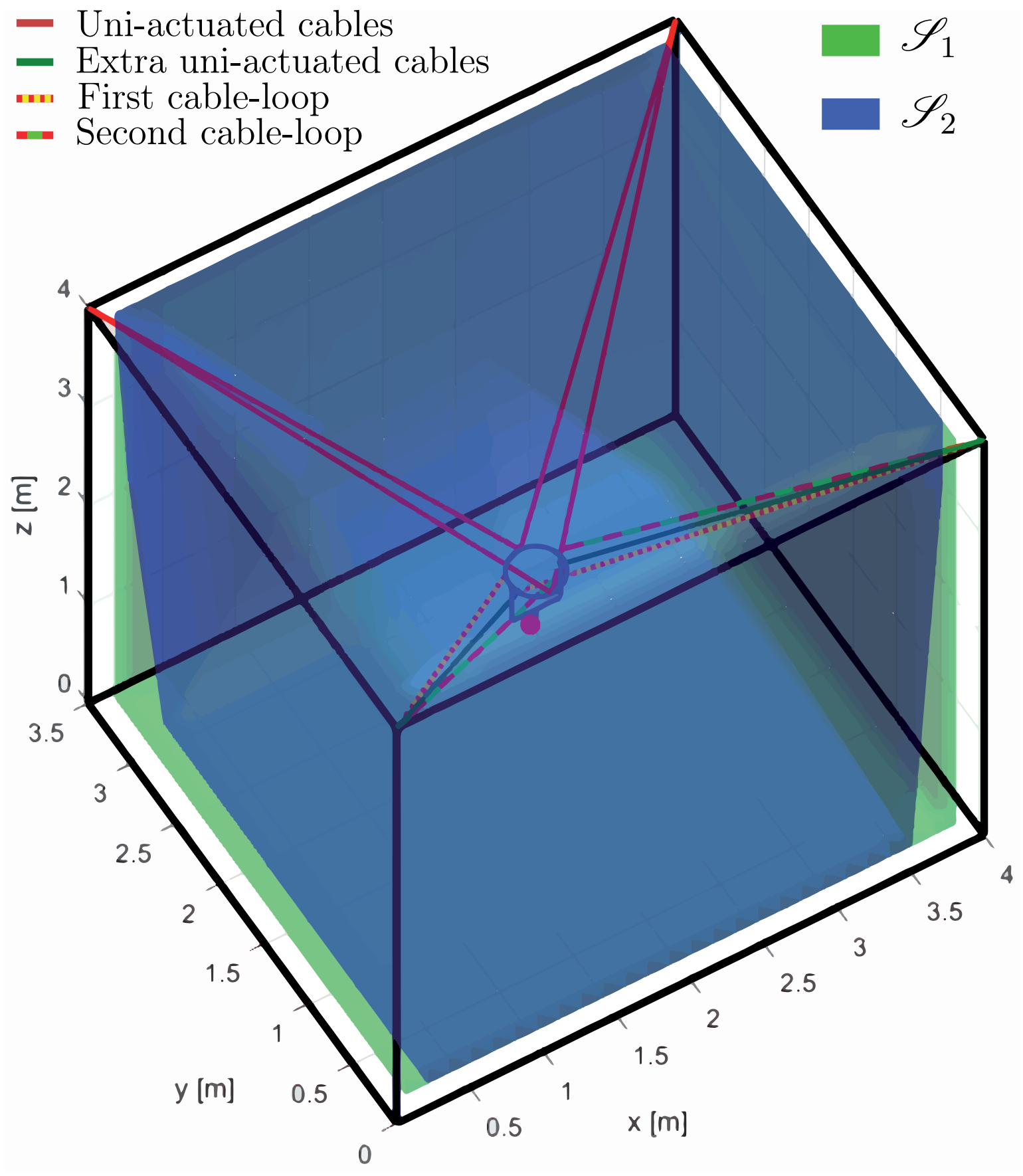

Fig. 11: Workspaces $\mathscr{S}_{1}$ and $\mathscr{S}_{2}$ associated with the optimum design of the CDPR with an embedded tilt-roll wrist with two additional uni-actuated cables

effects on the workspace size. It should be noted that, the considered workspace assumes no external forces on the movingplatform, except for gravity, so that the workspace analysis is relevant to applications such as, tomography scanning and surveillance.

The moving-platform is suspended by eight cables and its embedded tilt-roll wrist is driven through two bi-actuated cable-loops. As a result, the end-effector covers very large rotation amplitudes about two axes without singularities. This leads to a trade-off, however, between translational and rotational workspaces due to tension coupling in cable-loops for fully- 


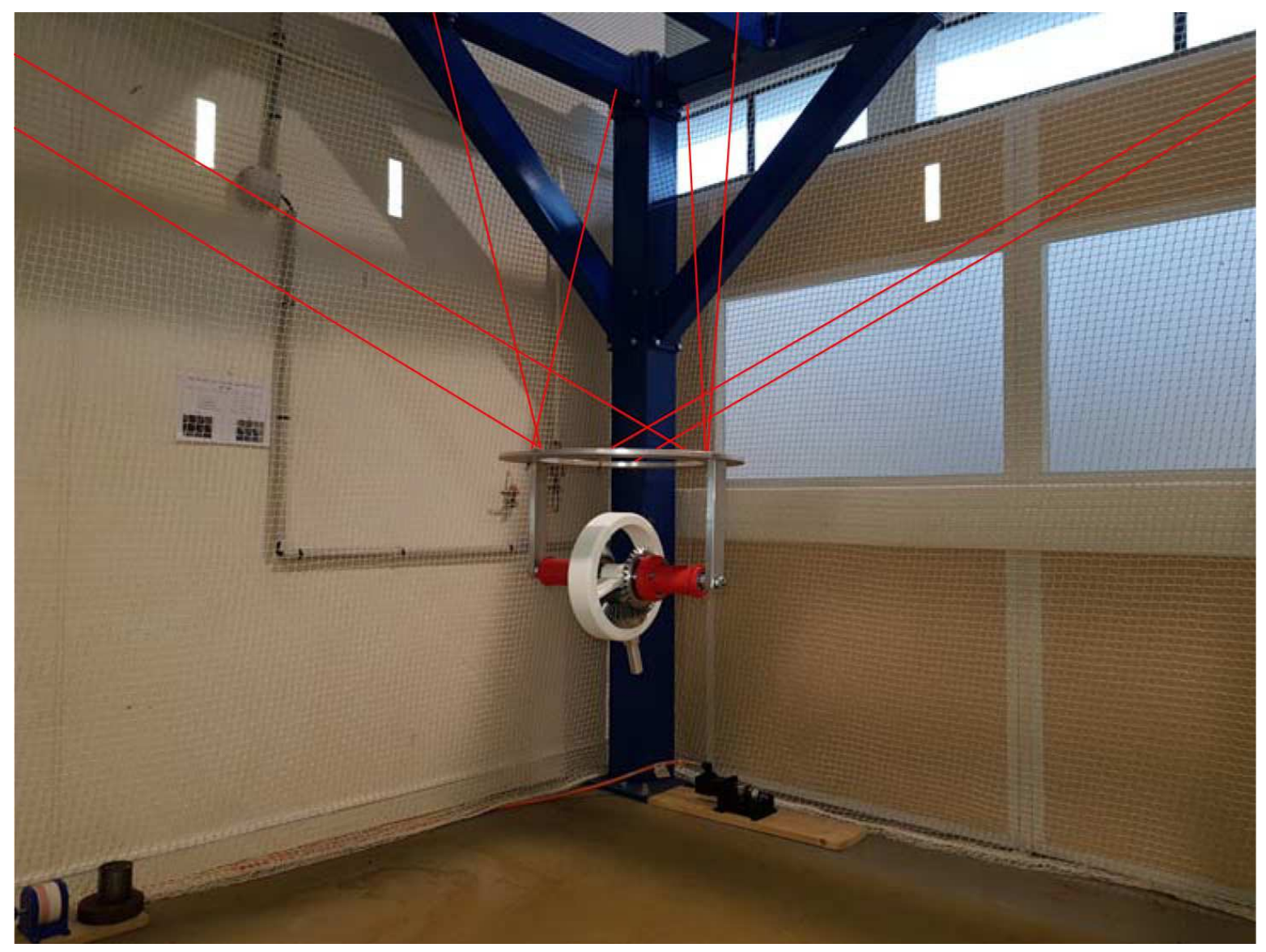

Fig. 12: Prototype of the CDPR with an embedded tilt-roll wrist

actuated CDPRs. Future work will consist in studying other factors influencing the size of the workspace, e.g., orientation of the moving-platform and over-actuation.

\section{Acknowledgments}

This work is supported by the RFI Altanstic 2020 in the framework of the CREATOR ${ }^{3}$ project. Jean-Christophe Ruel extracted the laser dot positions from the film of the experiments, and his help is dutifully acknowledged. Assistance provided by Marceau Métillon through the experimentation process is highly appreciated.

\section{Nomenclature}

CDPR: Cable-Driven Parallel Robot

DoF: Degree-of-Freedom

WFW: Wrench Feasible Workspace

$\mathscr{F}_{0}\left(O_{0}, x_{0}, y_{0}, z_{0}\right):$ Base frame centered at $O_{0}$

\footnotetext{
${ }^{3}$ robot parallèle à Câbles ayant un gRand Espace de trAvail en Translation et en ORientation
} 


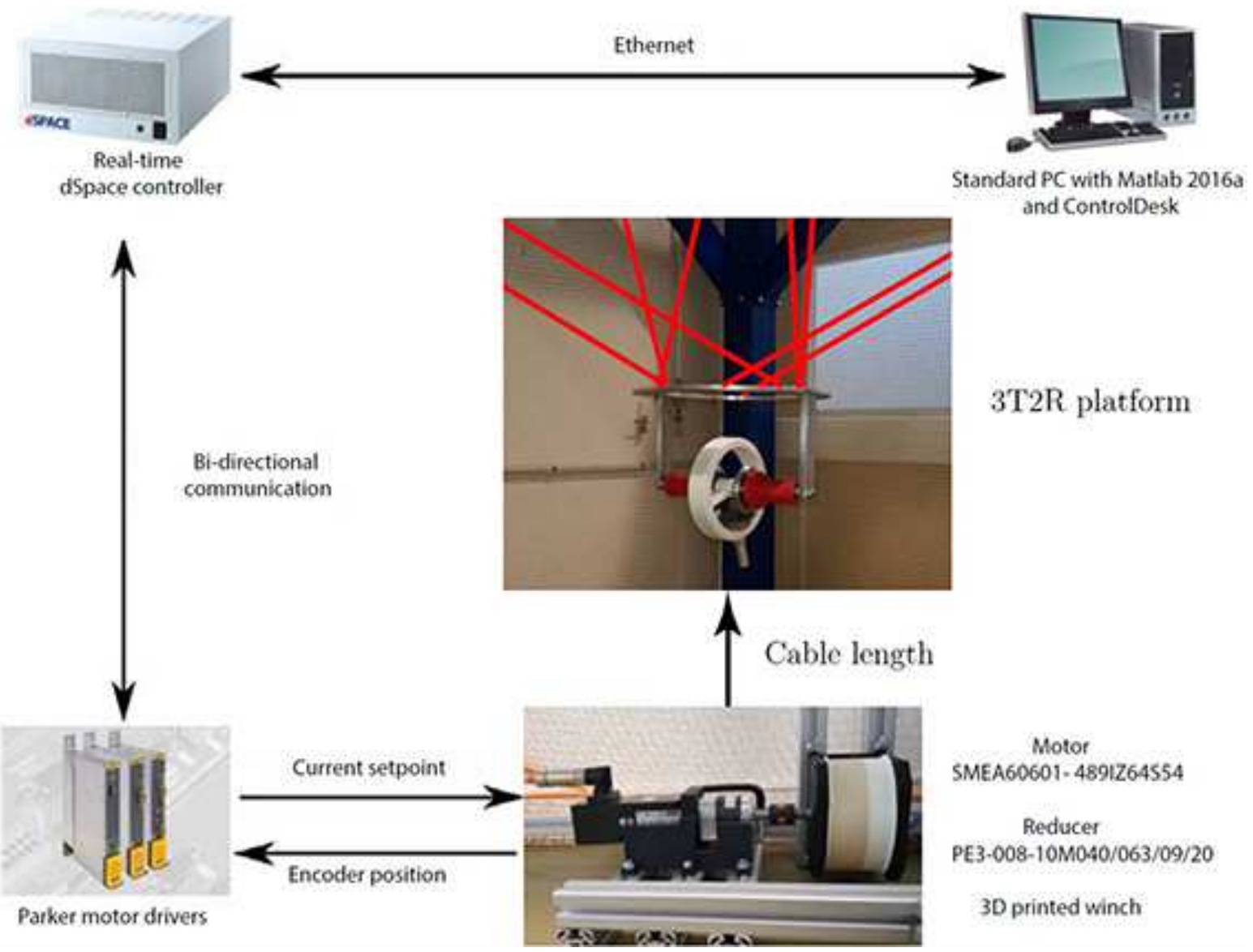

Fig. 13: Equivalent architecture of the prototype

$\mathscr{F}_{1}\left(P, x_{1}, y_{1}, z_{1}\right)$ : Coordinate frame attached to the moving-platform

$\mathscr{F}_{i}\left(O_{i}, x_{i}, y_{i}, z_{i}\right)$ : Coordinate frame attached to the part $\mathscr{P}_{i}, i=2, \ldots, 5$

$\mathscr{C}_{i}: i$-th cable of the manipulator, $i=1, \ldots, 8$

$\mathscr{C}_{12}:$ First cable-loop of the manipulator

$\mathscr{C}_{12}:$ Second cable-loop of the manipulator

$\mathscr{P}_{0}$ : Fixed frame of the manipulator

$\mathscr{P}_{1}$ : Structure of the moving-platform

$\mathscr{P}_{2}$ : Carrier of the tilt-roll wrist

$\mathscr{P}_{3}:$ First gear of the tilt-roll wrist

$\mathscr{P}_{4}$ : Second gear of the tilt-roll wrist

$\mathscr{P}_{5}$ : Terminal link of the tilt-roll wrist

$A_{i}: i$-th cable exit point $, i=1, \ldots, 8$

$B_{i}: i$-th cable anchor point, $i=1, \ldots, 8$ 


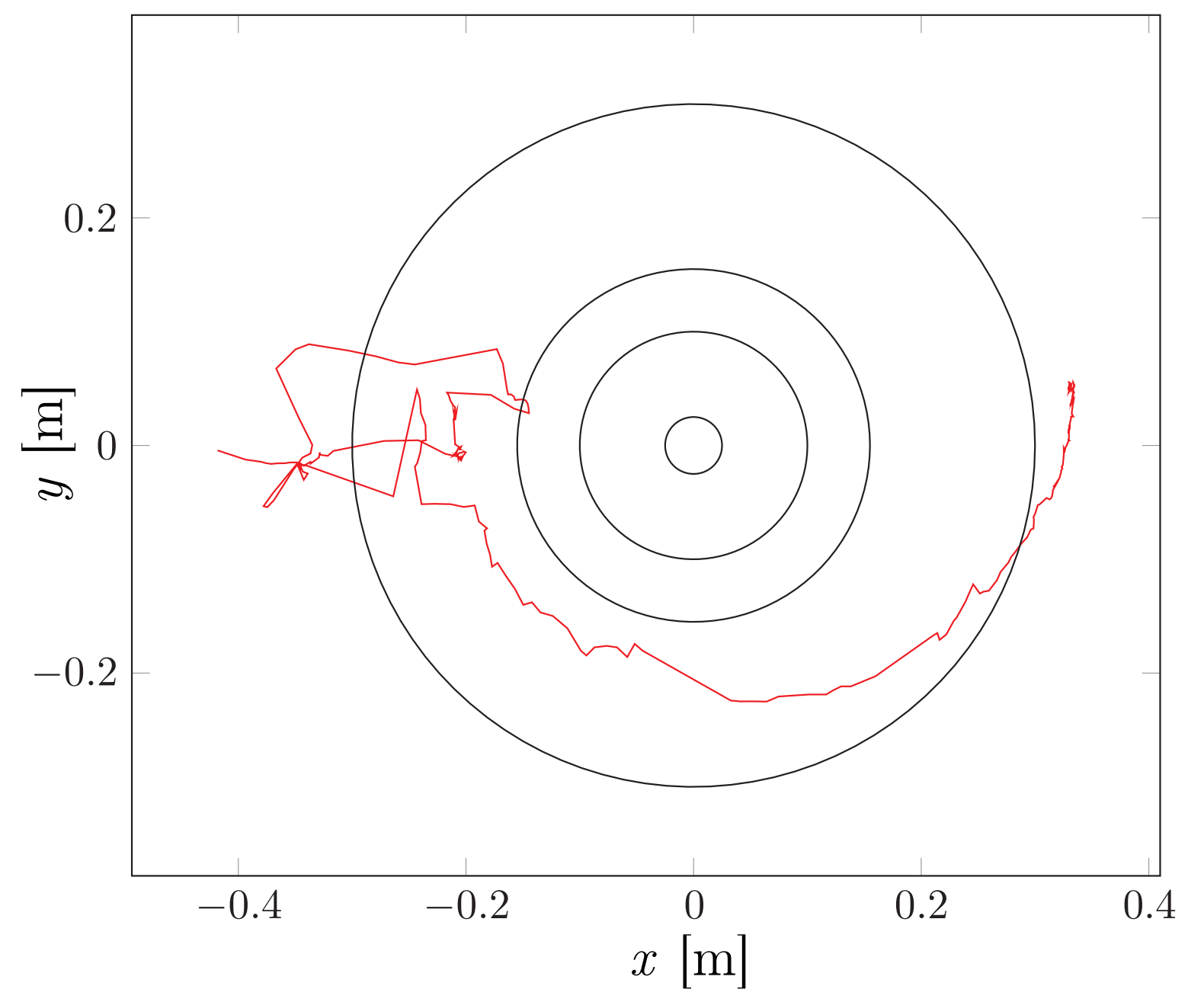

Fig. 14: Extracted laser dot trajectory during the second phase of the test-trajectory 2

$\mathbf{l}_{i}: i$-th cable vector pointing from point $B_{i}$ to point $A_{i}$

$l_{i}: i$-th cable length, $i=1, \ldots, 8$

$\mathbf{a}_{i}$ : Cartesian coordinates vector of point $A_{i}$

$\mathbf{b}_{i}$ : Cartesian coordinates vector of point $B_{i}$

${ }^{0} \mathbf{R}_{1}$ : Rotation matrix from frame $\mathscr{F}_{0}$ to frame $\mathscr{F}_{1}$

$\mathbf{u}_{i}: i$-th cable unit vector $i=1, \ldots, 8$

$\mathbf{t}_{i}: i$-th cable tension vector $i=1, \ldots, 8$

$t_{i}: i$-th cable tension, $i=1, \ldots, 8$

$m$ : Moving-platform mass

g: Gravitational acceleration vector

$g$ : Gravitational acceleration equal to $9.81 \mathrm{~m} / \mathrm{s}^{2}$

c: Cartesian coordinates vector of the CoM of the overall platform 


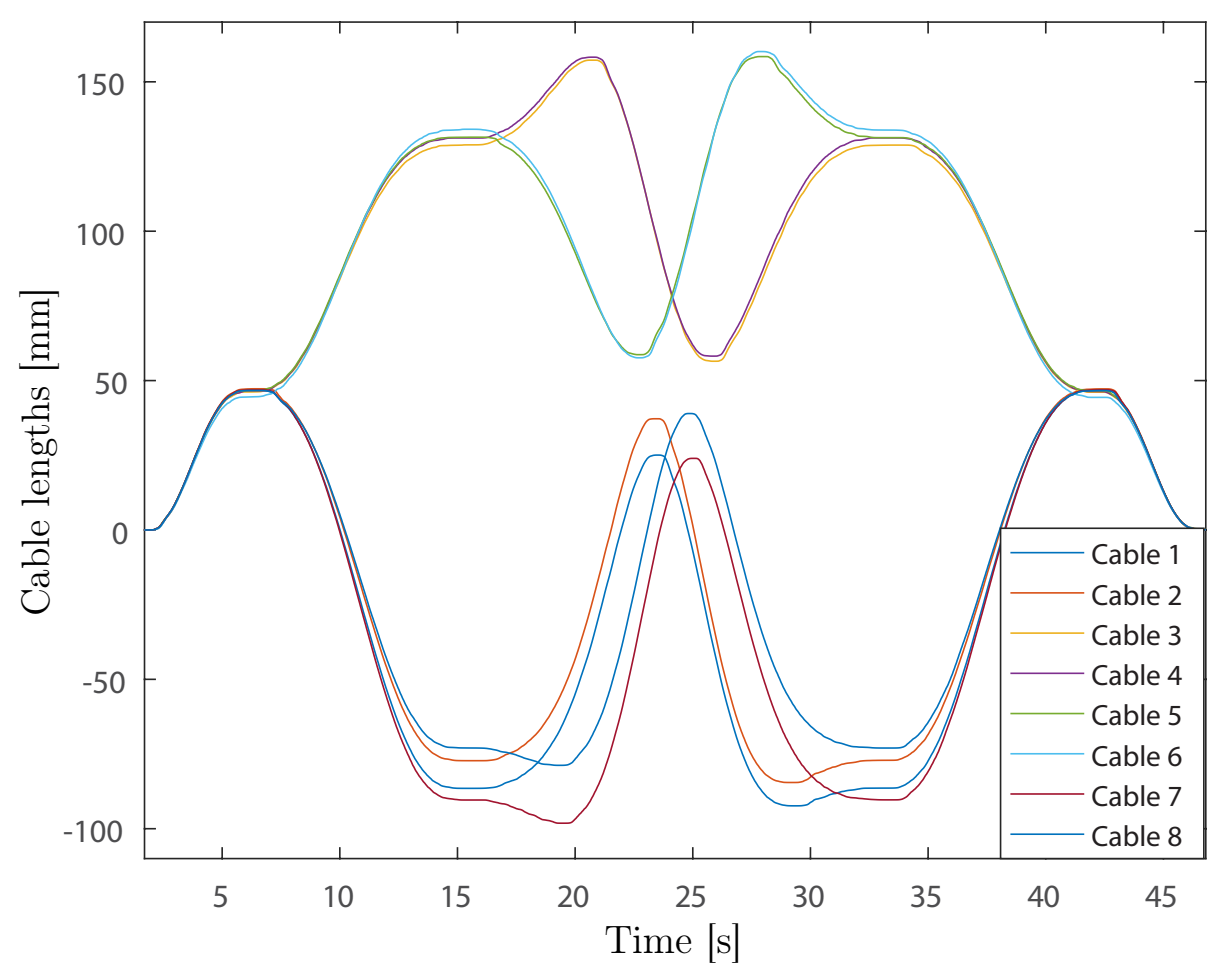

(a) Both the moving-platform and the wrist move

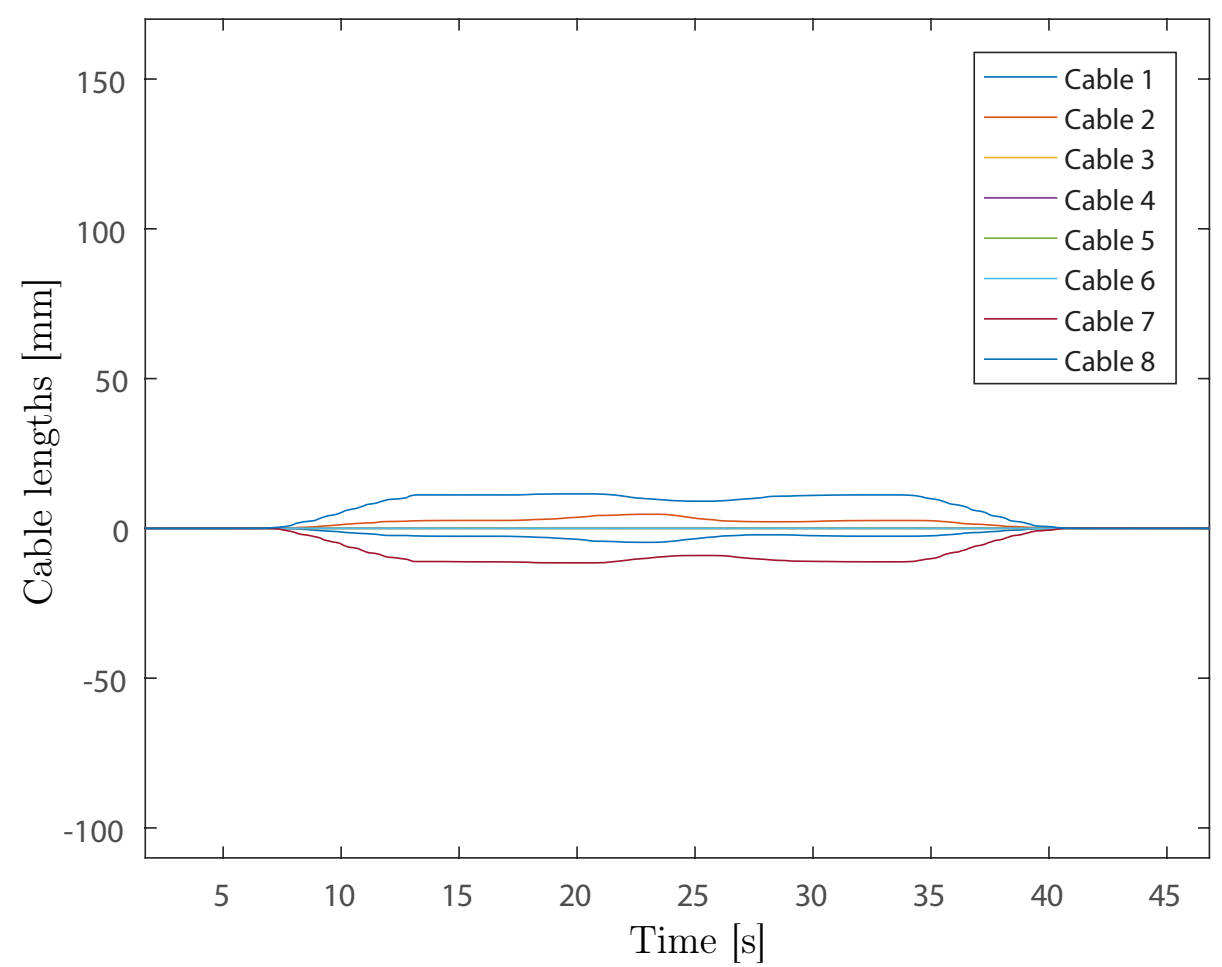

(b) Wrist motion only

Fig. 15: Cable length profiles for test-trajectory 2

$\mathbf{c}_{1}$ : Cartesian coordinates vector of the CoM of components $\mathscr{P}_{1}$ to $\mathscr{P}_{4}$

$\mathbf{c}_{2}$ : Cartesian coordinates vector of the CoM of $\mathscr{P}_{5}$ 


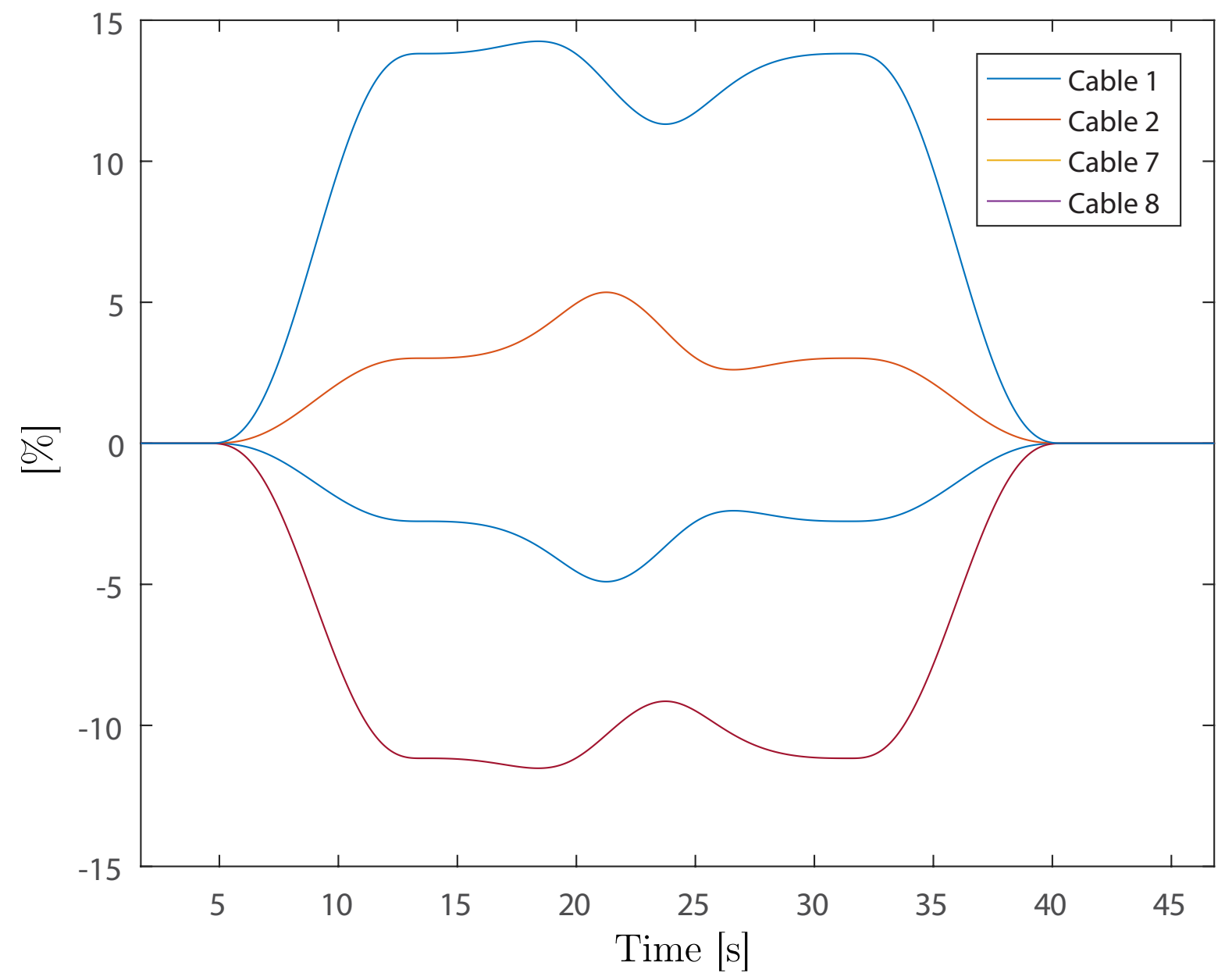

Fig. 16: Proportion of the cable-loop length due to the wrist motion during the test-trajectory 2

$m_{1}$ : Mass of components $\mathscr{P}_{1}$ to $\mathscr{P}_{4}$

$m_{2}$ : Mass of the end-effector

W: Wrench matrix

$\mathbf{d}_{i}$ : Moment exerted on the moving-platform by the $i$-th cable, $i=1, \ldots, 8$

$m_{b}$ : Mass of the moving-platform base

$m_{c}$ : Mass of the moving-platform columns

$m_{s}$ : Mass of the moving-platform shaft

$m_{\mathscr{P}_{i}}:$ Mass of the part $\mathscr{P}_{i}$

$\tau_{3}:$ Input tilt-roll wrist torque associated to $\mathscr{P}_{3}$

$\tau_{4}$ : Input tilt-roll wrist torque associated to $\mathscr{P}_{4}$

$r_{c}$ : Radius of the grooves made in $\mathscr{P}_{3}$ and $\mathscr{P}_{4}$

$\alpha$ : Tilt angle of the wrist 
$\beta$ : Roll angle of the wrist

$\mathbf{m}_{\alpha, \beta}=\left[m_{\alpha}, m_{\beta}\right]^{T}$ : The array of the moments applied by gravity onto the tilt-roll wrist about axes $z_{2}$ and $z_{5}$, respectively.

$\mathbf{t}_{\text {min }}$ : Cable tension lower-bound vector

$\mathbf{t}_{\max }$ : Cable tension upper-bound vector

$t_{\text {min }}:$ Minimum admissible cable tension

$t_{\text {max }}:$ Maximum admissible cable tension

p: Cartesian coordinates vector of point $P$

$h_{1}$ : Distance between $P$ and $O_{2}$ along $z$

$h_{2}$ : Distance between $\mathrm{O}_{2}$ and $\mathrm{C}_{2}$ along $z$

$w_{2}$ : Distance between $O_{2}$ and $C_{2}$ along $x$

$\mathbf{m}_{g}=\left[m_{g_{x}}, m_{g_{y}}, m_{g_{z}}\right]^{T}:$ Moment due to the weight of $\mathscr{P}_{5}$ exerted on the tilt-roll wrist

$\mathscr{T}:$ Cable tension space

$\mathbf{q}_{T R}=[\alpha, \beta]^{T}:$ Configuration of the tilt-roll wrist

$\mathscr{S}:$ Static workspace

$\mathscr{S}_{1}$ : Subset of the static workspace expressed in Eq. (22)

$\mathscr{S}_{2}$ : Subset of the static workspace expressed in Eq. (23)

$\mathscr{W}_{1}$ : Static workspace of an equivalent classical CDPR by replacing cable-loops with uni-actuated ones

$\mathscr{W}_{2}$ :wrench-feasible workspace of an equivalent classical CDPR by replacing cable-loops with uni-actuated ones, with the set of external wrenches due to the tilt-roll wrist motion defined in (23)

$N_{\mathscr{S}_{1}}$ : Number of points found to be inside the static workspace, $\mathscr{S}_{1}$

$N_{\mathscr{S}_{2}}:$ Number of points found to be inside the static workspace, $\mathscr{S}_{2}$

$\mathscr{R}_{\mathscr{S}_{i}}$ : Proportion of $\mathscr{S}_{1}$ to the task space

$\mathbf{x}$ : Decision variable vector

$\mathbf{x}_{l b}:$ Lower-bound of decision variable vector

$\mathbf{x}_{u b}:$ Upper-bound of decision variable vector

$r_{b}$ : Radius of the circle passing through $B_{i}, i=1, \ldots, 8$

$w_{b}$ : Width of the top-plate

$\theta_{i}$ : Angle defining the location of the $i$-th anchor point on the top-plate, $i=1, \ldots, 8$

$\theta_{c}$ : Angle defining the location of the anchor points of the cable-loops onto the top-plate 
$\theta_{u}$ : Angle defining the location of the anchor points of the uni-actuated cables onto the top-plate

$h_{e}$ : Length of the terminal link

$w_{e}$ : Width of the terminal link

$\mu$ : Gear ratio of the wrist

$\eta$ : Cable arrangement number

$\mathscr{N}_{a}$ : Total number of cable arrangements

$r_{i}$ : Pitch radius of the gear attached to part $\mathscr{P}_{i}, i=3,4,5$

$l_{0}$ : Length of the base frame, i.e., $\mathscr{P}_{0}$

$w_{0}$ : Width of the base frame, i.e., $\mathscr{P}_{0}$

$h_{0}$ : Height of the base frame, i.e., $\mathscr{P}_{0}$

$m_{\max }:$ Maximum admissible mass of the moving-platform

E: End-effector point

e: Cartesian coordinates vector of point $E$

\section{References}

[1] Lambert, C., Nahon, M., and Chalmers, D., 2007. "Implementation of an aerostat positioning system with cable control”. IEEE/ASME Transactions on Mechatronics, 12(1), Feb, pp. 32-40.

[2] Bosscher, P., Williams, R. L., Bryson, L. S., and Castro-Lacouture, D., 2007. "Cable-suspended robotic contour crafting system”. Automation in Construction, 17(1), pp. 45 - 55.

[3] Bostelman, R., Albus, J., Dagalakis, N., Jacoff, A., and Gross, J., 1994. "Applications of the nist robocrane". In Proceedings of the 5th International Symposium on Robotics and Manufacturing, Vol. 5.

[4] Gagliardini, L., Caro, S., Gouttefarde, M., and Girin, A., 2016. "Discrete reconfiguration planning for cable-driven parallel robots". Mechanism and Machine Theory, 100, pp. 313 - 337.

[5] Kawamura, S., Kino, H., and Won, C., 2000. "High-speed manipulation by using parallel wire-driven robots". Robotica, 18(1), pp. 13-21.

[6] Platis, A., Rasheed, T., Cardou, P., and Caro, S., 2018. Isotropic Design of the Spherical Wrist of a Cable-Driven Parallel Robot. Springer International Publishing, Cham, pp. 321-330.

[7] Khakpour, H., and Birglen, L., 2014. "Workspace augmentation of spatial 3-dof cable parallel robots using differential actuation”. In 2014 IEEE/RSJ International Conference on Intelligent Robots and Systems, pp. 3880-3885. 
[8] Khakpour, H., Birglen, L., and Tahan, S., 2014. "Synthesis of differentially driven planar cable parallel manipulators". IEEE Transactions on Robotics, 30(3), June, pp. 619-630.

[9] Khakpour, H., Birglen, L., and Tahan, S.-A., 2015. “Analysis and Optimization of a New Differentially Driven Cable Parallel Robot”. Journal of Mechanisms and Robotics, 7(3), 08. 034503.

[10] Lessanibahri, S., Cardou, P., and Caro, S., 2018. "Kinetostatic Analysis of a Simple Cable-Driven Parallel Crane". Vol. Volume 5A: 42nd Mechanisms and Robotics Conference of International Design Engineering Technical Conferences and Computers and Information in Engineering Conference. V05AT07A049.

[11] Lessanibahri, S., Cardou, P., and Caro, S., 2019. "Kinetostatic modeling of a cable-driven parallel robot using a tilt-roll wrist”. In Cable-Driven Parallel Robots, A. Pott and T. Bruckmann, eds., Springer International Publishing, pp. 109120.

[12] Guay, F., Cardou, P., Cruz-Ruiz, A. L., and Caro, S., 2014. "Measuring how well a structure supports varying external wrenches”. In New Advances in Mechanisms, Transmissions and Applications, V. Petuya, C. Pinto, and E.-C. Lovasz, eds., Springer Netherlands, pp. 385-392.

[13] Ruiz, A. L. C., Caro, S., Cardou, P., and Guay, F., 2015. "Arachnis: Analysis of robots actuated by cables with handy and neat interface software". In Cable-Driven Parallel Robots, A. Pott and T. Bruckmann, eds., Springer International Publishing, pp. 293-305.

[14] Erskine, J., Chriette, A., and Caro, S., 2019. "Wrench Analysis of Cable-Suspended Parallel Robots Actuated by Quadrotor Unmanned Aerial Vehicles”. Journal of Mechanisms and Robotics, 11(2), 02. 020909.

[15] Rasheed, T., Long, P., and Caro, S., 2019. "Wrench-Feasible Workspace of Mobile Cable-Driven Parallel Robots". Journal of Mechanisms and Robotics, 11, pp. 1-23.

[16] Bai, S. P., and Angeles, J., 2005. "The design of a gearless pitch-roll wrist". In Proceedings of the 2005 IEEE International Conference on Robotics and Automation, pp. 3213-3218.

[17] Baklouti, S., Courteille, E., Caro, S., and Dkhil, M., 2017. "Dynamic and Oscillatory Motions of Cable-Driven Parallel Robots Based on a Nonlinear Cable Tension Model”. Journal of Mechanisms and Robotics, 9(6), 10. 061014.

[18] Zake, Z., Chaumette, F., Pedemonte, N., and Caro, S., 2019. "Vision-based control and stability analysis of a cabledriven parallel robot”. IEEE Robotics and Automation Letters, 4(2), April, pp. 1029-1036.

[19] Hyodo, K., and Kobayashi, H., 1992. "Kinematic and control issues on tendon controlled wrist mechanism”. Journal of the Robotics Society of Japan, 10(6), pp. 809-816. 


\section{List of Figures}

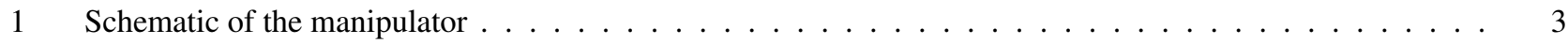

2 Schematic of the moving-platform with an embedded tilt-roll wrist $\ldots \ldots \ldots \ldots \ldots$

3 Section-view of the moving-platform and the tilt-roll wrist $\ldots \ldots \ldots \ldots \ldots$

4 Top-view of the base frame and up-scaled moving-platform with the embedded tilt-roll wrist $\ldots \ldots \ldots$

$5 \quad$ Workspaces $\mathscr{S}_{1}$ and $\mathscr{S}_{2}$ associated with the optimum design of the CDPR with an embedded tilt-roll wrist . 17

$6 \quad$ Ratio $\mathscr{R}_{\mathscr{S}_{2}}$ of static workspace $\mathscr{S}_{2}$ as a function of $m_{1}$ and $m_{2} \ldots \ldots \ldots \ldots$

$7 \quad$ Ratio $\mathscr{R}_{\mathscr{S}_{2}}$ of static workspace $\mathscr{S}_{2}$ as a function of $\theta_{c}$ and $r_{b} \ldots \ldots \ldots \ldots$

$8 \quad$ Ratio $\mathscr{R}_{\mathscr{S}_{2}}$ of static workspace $\mathscr{S}_{2}$ as a function of $\theta_{c}$ and $\theta_{u} \ldots \ldots \ldots \ldots$

9 Ratio $\mathscr{R}_{\mathscr{S}_{2}}$ of static workspace $\mathscr{S}_{2}$ as a function of $\theta_{u}$ and $r_{b} \ldots \ldots \ldots \ldots \ldots \ldots \ldots \ldots$

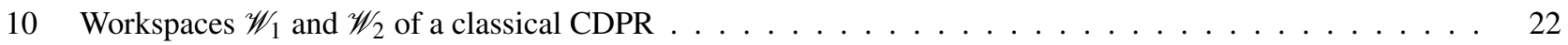

11 Workspaces $\mathscr{S}_{1}$ and $\mathscr{S}_{2}$ of the hybrid CDPR with two additional uni-actuated cables $\ldots \ldots \ldots$

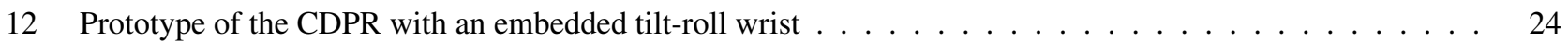

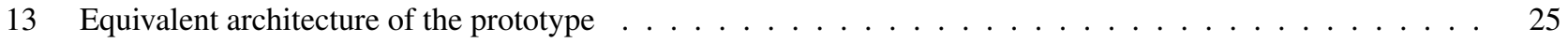

14 Extracted laser dot trajectory during the second phase of the test-trajectory $2 \ldots \ldots \ldots$

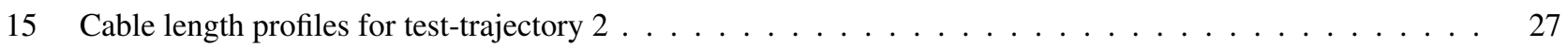

16 Proportion of the cable-loop length due to the wrist motion during the test-trajectory $2 \ldots \ldots 28$ 


\section{List of Tables}

1 Boundaries and optimum values of the design variables $\ldots \ldots \ldots \ldots \ldots \ldots$

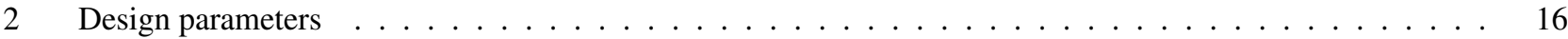

3 Tuning parameters of the genetic algorithm $\ldots \ldots \ldots \ldots \ldots \ldots \ldots \ldots \ldots \ldots$ 